Article

\title{
Riemann-Liouville Fractional Integral Inequalities for Generalized Pre-Invex Functions of Interval-Valued Settings Based upon Pseudo Order Relation
}

\author{
Muhammad Bilal Khan ${ }^{1}\left(\mathbb{D}\right.$, Hatim Ghazi Zaini ${ }^{2}$, Savin Treanță ${ }^{3} \mathbb{D}$, Mohamed S. Soliman ${ }^{4}$ (D) \\ and Kamsing Nonlaopon $5, *$ (D) \\ 1 Department of Mathematics, COMSATS University Islamabad, Islamabad 45550, Pakistan; \\ bila142742@gmail.com \\ 2 Department of Computer Science, College of Computers and Information Technology, Taif University, \\ P.O. Box 11099, Taif 21944, Saudi Arabia; h.zaini@tu.edu.sa \\ 3 Department of Applied Mathematics, University Politehnica of Bucharest, 060042 Bucharest, Romania; \\ savin.treanta@upb.ro \\ 4 Department of Electrical Engineering, College of Engineering, Taif University, P.O. Box 11099, \\ Taif 21944, Saudi Arabia; soliman@tu.edu.sa \\ 5 Department of Mathematics, Faculty of Science, Khon Kaen University, Khon Kaen 40002, Thailand \\ * Correspondence: nkamsi@kku.ac.th; Tel.: +66-866-421-582
}

check for

updates

Citation: Khan, M.B.; Zaini, H.G.;

Treanță, S.; Soliman, M.S.;

Nonlaopon, K. Riemann-Liouville Fractional Integral Inequalities for Generalized Pre-Invex Functions of Interval-Valued Settings Based upon Pseudo Order Relation. Mathematics 2022, 10, 204. https://doi.org/ $10.3390 /$ math10020204

Academic Editor: Sitnik Sergey

Received: 7 December 2021

Accepted: 7 January 2022

Published: 10 January 2022

Publisher's Note: MDPI stays neutral with regard to jurisdictional claims in published maps and institutional affiliations.

Copyright: (c) 2022 by the authors. Licensee MDPI, Basel, Switzerland. This article is an open access article distributed under the terms and conditions of the Creative Commons Attribution (CC BY) license (https:// creativecommons.org/licenses/by/ $4.0 /)$.

\begin{abstract}
The concepts of convex and non-convex functions play a key role in the study of optimization. So, with the help of these ideas, some inequalities can also be established. Moreover, the principles of convexity and symmetry are inextricably linked. In the last two years, convexity and symmetry have emerged as a new field due to considerable association. In this paper, we study a new version of interval-valued functions $(I-V \cdot F s)$, known as left and right $\chi$-pre-invex interval-valued functions (LR- $\chi$-pre-invex $I-V \cdot F s)$. For this class of non-convex $I-V \cdot F s$, we derive numerous new dynamic inequalities interval Riemann-Liouville fractional integral operators. The applications of these repercussions are taken into account in a unique way. In addition, instructive instances are provided to aid our conclusions. Meanwhile, we'll discuss a few specific examples that may be extrapolated from our primary findings.
\end{abstract}

Keywords: LR- $\chi$-pre-invex interval-valued function; interval Riemann-Liouville fractional integral operator; Hermite-Hadamard inequality; Hermite-Hadamard Fejér inequality

\section{Introduction}

The Hermite-Hadamard inequality (see [1,2], p. 137) is a well-known inequality in convex function theory, with a geometrical explanation and a wide range of applications. Hermite-Hadamard inequality ( $\mathrm{H}-\mathrm{H}$ inequality) is a development of the concept of convexity, and it logically follows from Jensen's inequality. In recent years, the $H-H$ inequality for convex functions has sparked a lot of attention, and several refinements and extensions have been investigated; see [3-14] and the references therein.

On the other hand, interval analysis is a subset of set-valued analysis and is concerned with the study of intervals in the context of mathematical analysis and topology. It was developed as a means of dealing with interval uncertainty, which is included in many mathematical or computer models of deterministic real-world systems. A historical example of an interval enclosure is Archimedes' method for calculating the circumference of a circle. In 1966, Moore [15] released the first book on interval analysis. Moore is recognized as being the first usage of intervals in computer mathematics. Following the release of his book, a lot of scientists began to study interval arithmetic's theory and applications. Because of its universality, interval analysis is currently a useful approach in a range of sectors that are interested in ambiguous data. Moreover, interval analysis has also applications in 
different fields like in error analysis, computer graphics, error analysis, experimental and computational physics, and many more.

Numerous significant inequalities for $I-V \cdot F s$ (Hermite-Hadamard, Ostrowski, etc.) have been investigated in recent years. In [16,17], Chalco-Cano et al. constructed Ostrowski type inequalities for $I-V \cdot F s$ using the Hukuhara derivative for $I-V \cdot F s$. Román-Flores et al. developed Minkowski and Beckenbach's inequality for $I-V \cdot F s$ in [18]. For more information, see [18-22] and the references therein. Moreover, inequalities can be examined for the more general set-valued mappings for example, Sadowska [23] introduced the $H-H$ inequality general set-valued mappings. Similarly, for generalized inequalities, we refer to the following articles, see [24,25] and the references therein. Recently, Khan et al. extended the interval $\mathrm{H}-\mathrm{H}$ inequalities in terms of fuzzy interval $\mathrm{H}-\mathrm{H}$ inequalities using fuzzy Riemannian and fuzzy Riemann-Liouville fractional integral operators such as in [26]. Khan et al. also presented the new class of convex fuzzy mappings known as $\left(\chi_{1}, \chi_{2}\right)$-convex fuzzy-interval-valued functions $\left(\left(\chi_{1}, \chi_{2}\right)\right.$-convex $\left.F-I-V \cdot F\right)$ and obtained the new version of $H-H$ inequalities for $\left(\chi_{1}, \chi_{2}\right)$-convex F-I-V.Fs. Moreover, Khan et al. introduced new notions of generalized convex $F-I-V \cdot F s$, and derived new fractional $H-H$ type and $H-H$ type inequalities for convex $F-I-V \cdot F s$ [27-32]. For more analysis and applications of $F-I-V \cdot F s$, see [33-50] and the references therein.

This study is organized as follows: Section 2 presents preliminary and new concepts and results in interval space, and convex analysis. Section 3 obtains interval $H-H$ inequalities and $H-H$ Fejér inequalities for LR- $\chi$-pre-invex $I-V$-Fs via interval Riemann-Liouville fractional integral operators. In addition, some interesting examples are also given to verify our results. Section 4 gives conclusions and future plans.

\section{Preliminaries}

Let $\mathcal{K}_{C}$ stand for the collection of all closed and bounded intervals of $\mathbb{R}$. We use $\mathcal{K}_{C}^{+}$to represent the set of all positive intervals. The collections of all Riemann integrable real valued functions and Riemann integrable $I-V$-F are denoted by $\mathcal{R}_{[\mu, \omega]}$ and $\mathcal{I} \mathcal{R}_{[\mu, \omega]}$, respectively. For more conceptions on $I-V \cdot F s$, see [36]. Moreover, we have:

Remark 1. [35] (i) The relation " $\leq_{p}$ " defined on $\mathcal{K}_{C}$ by:

$$
\left[\mathfrak{U}_{*}, \mathfrak{U}^{*}\right] \leq_{p}\left[\mathcal{Z}_{*}, \mathcal{Z}^{*}\right] \text { if and only if } \mathfrak{U}_{*} \leq \mathcal{Z}_{*}, \mathfrak{U}^{*} \leq \mathcal{Z}^{*},
$$

for all $\left[\mathfrak{U}_{*}, \mathfrak{U}^{*}\right],\left[\mathcal{Z}_{*}, \mathcal{Z}^{*}\right] \in \mathcal{K}_{C}$, it is a pseudo-order relation. For given $\left[\mathfrak{U}_{*}, \mathfrak{U}^{*}\right],\left[\mathcal{Z}_{*}, \mathcal{Z}^{*}\right] \in \mathcal{K}_{C}$, we say that $\left[\mathfrak{U}_{*}, \mathfrak{U}^{*}\right] \leq_{p}\left[\mathcal{Z}_{*}, \mathcal{Z}^{*}\right]$ if and only if $\mathfrak{U}_{*} \leq \mathcal{Z}_{*}, \mathfrak{U}^{*} \leq \mathcal{Z}^{*}$ or $\mathfrak{U}_{*} \leq \mathcal{Z}_{*}, \mathfrak{U}^{*}<\mathcal{Z}^{*}$. The relation $\left[\mathfrak{U}_{*}, \mathfrak{U}^{*}\right] \leq_{p}\left[\mathcal{Z}_{*}, \mathcal{Z}^{*}\right]$ coincident to $\left[\mathfrak{U}_{*}, \mathfrak{U}^{*}\right] \leq\left[\mathcal{Z}_{*}, \mathcal{Z}^{*}\right]$ on $\mathcal{K}_{C}$.

(ii) It can be easily seen that " $\leq_{p}$ " looks like "left and right" on the real line $\mathbb{R}$, so we call " $\leq_{p}$ " "left and right" (or "LR" order, in short).

The concept of the Riemann integral for $I-V-F$ first introduced by Moore [15] is defined as follows:

Theorem 1. [15] If $\mathfrak{S}:[\mu, \omega] \subset \mathbb{R} \rightarrow \mathcal{K}_{C}$ is an I-V.Fon $[\mu, \omega]$ such that $\mathfrak{S}(x)=\left[\mathfrak{S}_{*}(x), \mathfrak{S}^{*}(x)\right]$, then $\mathfrak{S}$ is Riemann integrable over $[\mu, \omega]$ if and only if, $\mathfrak{S}_{*}(x)$ and $\mathfrak{S}^{*}(x)$ both are Riemann integrable over $[\mu, \omega]$ such that:

$$
(I R) \int_{\mu}^{\omega} \mathfrak{S}(x) d x=\left[(R) \int_{\mu}^{\omega} \mathfrak{S}_{*}(x) d x,(R) \int_{\mu}^{\omega} \mathfrak{S}^{*}(x) d x\right]
$$

Lupulescu and Budak et al. [36,37] introduced the following interval Riemann-Liouville fractional integral operators: 
Let $\alpha>0$ and $L\left([\mu, \omega], \mathcal{K}_{C}^{+}\right)$be the collection of all Lebesgue measurable I-V-Fs on $[\mu, \omega]$. Then the interval left and right Riemann-Liouville fractional integrals of $\mathfrak{S} \in L\left([\mu, \omega], \mathcal{K}_{C}^{+}\right)$with order $\alpha>0$ are defined by:

$$
\mathcal{I}_{\mu^{+}}^{\alpha} \mathfrak{S}(x)=\frac{1}{\Gamma(\alpha)} \int_{\mu}^{x}(x-\varsigma)^{\alpha-1} \mathfrak{S}(\varsigma) d \varsigma, \quad(x>\mu),
$$

and:

$$
\mathcal{I}_{\omega^{-}}^{\alpha} \mathfrak{S}(x)=\frac{1}{\Gamma(\alpha)} \int_{x}^{\omega}(\varsigma-x)^{\alpha-1} \mathfrak{S}(\varsigma) d \varsigma, \quad(x<\omega)
$$

respectively, where $\Gamma(x)=\int_{0}^{\infty} \varsigma^{x-1} e^{-\varsigma} d \varsigma$ is the Euler gamma function.

Definition 1. [34] A real-valued function $\mathfrak{S}:[\mu, \omega] \rightarrow \mathbb{R}^{+}$is named as convex function if:

$$
\mathfrak{S}(\varsigma x+(1-\varsigma) z) \leq \varsigma \mathfrak{S}(x)+(1-\varsigma) \mathfrak{S}(z),
$$

for all $x, z \in[\mu, \omega], \varsigma \in[0,1]$. If (5) is reversed, then $\mathfrak{S}$ is named as concave.

Definition 2. [40] A real valued function $\mathfrak{S}:[\mu, \omega] \rightarrow \mathbb{R}^{+}$is named as pre-invex function if:

$$
\mathfrak{S}(x+(1-\varsigma) \varphi(z, x)) \leq \varsigma \mathfrak{S}(x)+(1-\varsigma) \mathfrak{S}(z),
$$

for all $x, z \in[\mu, \omega], \varsigma \in[0,1]$, where $\varphi:[\mu, \omega] \times[\mu, \omega] \rightarrow \mathbb{R}$. If (6) is reversed, then $\mathfrak{S}$ is named as pre-incave.

Definition 3. [35] The I-V.F $\mathfrak{S}:[\mu, \omega] \rightarrow \mathcal{K}_{C}^{+}$is named as LR-convex I-V.F on $[\mu, \omega]$ if:

$$
\mathfrak{S}(\varsigma x+(1-\varsigma) z) \leq_{p} \varsigma \mathfrak{S}(x)+(1-\varsigma) \mathfrak{S}(z),
$$

for all $x, z \in[\mu, \omega], \varsigma \in[0,1]$. If (7) is reversed, then $\mathfrak{S}$ is named as LR-concave I-V.F on $[\mu, \omega]$. $\mathfrak{S}$ is affine, if and only if it is both LR-convex and LR-concave I-V.F.

Definition 4. [41] The I-V.F $\mathfrak{S}:[\mu, \omega] \rightarrow \mathcal{K}_{C}^{+}$is named as LR-pre-invex I-V.F on invex interval $[\mu, \omega]$ if:

$$
\mathfrak{S}(x+(1-\varsigma) \varphi(z, x)) \leq_{p} \varsigma \mathfrak{S}(x)+(1-\varsigma) \mathfrak{S}(z),
$$

for all $x, z \in[\mu, \omega], \varsigma \in[0,1]$, where $\varphi:[\mu, \omega] \times[\mu, \omega] \rightarrow \mathbb{R}$. If (8) is reversed then, $\mathfrak{S}$ is named as $L R$-pre-incave I-V.F on $[\mu, \omega] . \mathfrak{S}$ is a $L R$-affine if and only if, it is both $L R$-pre-invex and $L R$-pre-incave $I-V \cdot F s$.

Definition 5. Let $\chi:[0,1] \subseteq[\mu, \omega] \rightarrow \mathbb{R}^{+}$such that $\chi 0$. Then, I-V.F $\mathfrak{S}:[\mu, \omega] \rightarrow \mathcal{K}_{C}^{+}$is said to be $L R$ - $\chi$-pre-invex $I-V \cdot F$ on $[\mu, \omega]$ if:

$$
\mathfrak{S}(x+(1-\varsigma) \varphi(x, z)) \leq_{p} \chi(\varsigma) \mathfrak{S}(x)+\chi(1-\varsigma) \mathfrak{S}(z),
$$

for all $x, z \in[\mu, \omega], \varsigma \in[0,1]$, where $\varphi:[\mu, \omega] \times[\mu, \omega] \rightarrow \mathbb{R}$. If $\mathfrak{S}$ is $L R$ - $\chi$-pre-incave on $[\mu, \omega]$, then inequality (9) is reversed.

Remark 2. If $\chi(\varsigma)=\varsigma$, then LR- $\chi$-pre-invex I-V.F becomes LR-pre-invex I-V.F. If $\chi(\varsigma) \equiv 1$, then $L R$ - $\chi$-pre-invex I-V.F becomes LR-P I-V.F, that is:

$$
\mathfrak{S}(x+(1-\varsigma) \varphi(x, z)) \leq{ }_{p} \mathfrak{S}(x)+\mathfrak{S}(z), \forall x, z \in[\mu, \omega], \mathfrak{s} \in[0,1] .
$$


Theorem 2. Let $\chi:[0,1] \subseteq[\mu, \omega] \rightarrow \mathbb{R}$ be a non-negative real valued function such that $\chi 0$ and let $\mathfrak{S}:[\mu, \omega] \rightarrow \mathcal{K}_{C}^{+}$be a I-V.F such that:

$$
\mathfrak{S}(z)=\left[\mathfrak{S}_{*}(z), \mathfrak{S}^{*}(z)\right]
$$

for all $z \in[\mu, \omega]$. Then, $\mathfrak{S}(z)$ is LR- $\chi$-pre-invex $I-V \cdot F$ on $[\mu, \omega]$, if and only if, $\mathfrak{S}_{*}(z)$ and $\mathfrak{S}^{*}(z)$ both are $\chi$-pre-invex.

Proof. Assume that, $\mathfrak{S}_{*}(x)$ and $\mathfrak{S}^{*}(x)$ are $\chi$-pre-invex on $[\mu, \omega]$. Then from (6), we have:

$$
\mathfrak{S}_{*}(x+(1-\varsigma) \varphi(x, z)) \leq \chi(\varsigma) \mathfrak{S}_{*}(x)+\chi(1-\varsigma) \mathfrak{S}_{*}(z), \forall x, z \in[\mu, \omega], \varsigma \in[0,1],
$$

and:

$$
\mathfrak{S}^{*}(x+(1-\varsigma) \varphi(x, z)) \leq \chi(\varsigma) \mathfrak{S}^{*}(x)+\chi(1-\varsigma) \mathfrak{S}^{*}(z), \forall x, z \in[\mu, \omega], \varsigma \in[0,1] .
$$

Then by (11), we obtain:

$$
\begin{aligned}
\mathfrak{S}(x+(1-\varsigma) \varphi(x, z)) & =\left[\mathfrak{S}_{*}(x+(1-\varsigma) \varphi(x, z)), \mathfrak{S}^{*}(x+(1-\varsigma) \varphi(x, z))\right] \\
& \leq\left[\chi(\varsigma) \mathfrak{S}_{*}(x), \chi(\varsigma) \mathfrak{S}^{*}(x)\right]+\left[\chi(1-\varsigma) \mathfrak{S}_{*}(z), \chi(1-\varsigma) \mathfrak{S}^{*}(z)\right]
\end{aligned}
$$

that is:

$$
\mathfrak{S}(x+(1-\varsigma) \varphi(x, z)) \leq_{p} \chi(\varsigma) \mathfrak{S}(x)+\chi(1-\varsigma) \mathfrak{S}(z), \forall x, z \in[\mu, \omega], \varsigma \in[0,1] .
$$

Hence, $\mathfrak{S}$ is LR- $\chi$-pre-invex IVF on $[\mu, \omega]$.

Conversely, let $\mathfrak{S}$ be a LR- $\chi$-pre-invex IVF on $[\mu, \omega]$. Then for all $x, z \in[\mu, \omega]$ and $\varsigma \in[0,1]$, we have:

$$
\mathfrak{S}(x+(1-\varsigma) \varphi(x, z)) \leq_{p} \chi(\varsigma) \mathfrak{S}(x)+\chi(1-\varsigma) \mathfrak{S}(z)
$$

Therefore, from (11), we have:

$$
\mathfrak{S}(x+(1-\varsigma) \varphi(x, z))=\left[\mathfrak{S}_{*}(x+(1-\varsigma) \varphi(x, z)), \mathfrak{S}^{*}(x+(1-\varsigma) \varphi(x, z))\right] .
$$

Again, from (11), we obtain:

$\chi(\varsigma) \mathfrak{S}(x)+\chi(1-\varsigma) \mathfrak{S}(x)=\left[\chi(\varsigma) \mathfrak{S}_{*}(x), \chi(\varsigma) \mathfrak{S}^{*}(x)\right]+\left[\chi(1-\varsigma) \mathfrak{S}_{*}(z), \chi(1-\varsigma) \mathfrak{S}^{*}(z)\right]$ for all $x, z \in[\mu, \omega]$ and $\varsigma \in[0,1]$. Then by LR- $\chi$-pre-invexity of $\mathfrak{S}$, we have for all $x, z \in[\mu, \omega]$ and $\varsigma \in[0,1]$ such that:

$$
\mathfrak{S}_{*}(x+(1-\varsigma) \varphi(x, z)) \leq \chi(\varsigma) \mathfrak{S}_{*}(x)+\chi(1-\varsigma) \mathfrak{S}_{*}(z),
$$

and:

$$
\mathfrak{S}^{*}(x+(1-\varsigma) \varphi(x, z)) \leq \chi(\varsigma) \mathfrak{S}^{*}(x)+\chi(1-\varsigma) \mathfrak{S}^{*}(z),
$$

hence, the result follows.

Example 1. We consider $\chi(\varsigma)=\varsigma$, for $\varsigma \in[0,1]$ and the $I-V \cdot F \mathfrak{S}:[0,4] \rightarrow \mathcal{K}_{C}^{+}$defined by $\mathfrak{S}(z)=\left[z, 2 e^{z^{2}}\right]$. Since end point functions $\mathfrak{S}_{*}(z), \mathfrak{S}^{*}(z)$ are $\chi$-pre-invex functions with respect to $\varphi(x, z)=x-z$. Hence $\mathfrak{S}(z)$ is $L R-\chi$-pre-invex $I-V \cdot F$.

Remark 3. If $\chi(\varsigma) \equiv \varsigma$ and $\mathfrak{S}_{*}(z)=\mathfrak{S}^{*}(z)$, then from (8), we obtain the inequality (6).

If $\chi(\varsigma) \equiv \varsigma$ and $\mathfrak{S}_{*}(z)=\mathfrak{S}^{*}(z)$ and $\varphi(x, z)=x-z$, then from (8), we obtain the inequality (5). 
We'll need to make the following assumption about the function $\varphi:[\mu, \omega] \times[\mu, \omega] \rightarrow \mathbb{R}$, which will be crucial in the major findings.

Condition C. [40]

$$
\begin{gathered}
\varphi(x, z+\varsigma \varphi(x, z))=(1-\varsigma) \varphi(x, z), \\
\varphi(z, z+\varsigma \varphi(x, z))=-\varsigma \varphi(x, z) .
\end{gathered}
$$

Note that $\forall z, x \in[\mu, \omega]$ and $\varsigma \in[0,1]$, then from condition $C$ we have

$$
\varphi\left(z+\varsigma_{2} \varphi(x, z), z+\varsigma_{1} \varphi(x, z)\right)=\left(\varsigma_{2}-\varsigma_{1}\right) \varphi(x, z)
$$

Clearly for $\varsigma=0$, we have $\varphi(x, z)=0$ if and only if $x=z$, for all $z, x \in[\mu, \omega]$. For the application of condition $C$, see $[40,41]$.

\section{Interval Fractional Hermite-Hadamard Inequalities}

In this section, we will prove some new $H-H$ type inequalities for LR- $\chi$-pre-invex $I-V \cdot F S$ via Riemann-Liouville fractional integral operators. In the next results, we will denote $L\left([\mu, \mu+\varphi(\omega, \mu)], \mathcal{K}_{C}^{+}\right)$as the family of Lebesgue measurable $I-V \cdot F s$.

Theorem 3. Let $\mathfrak{S}:[\mu, \mu+\varphi(\omega, \mu)] \rightarrow \mathcal{K}_{C}^{+}$be a LR--pre-invex $I-V \cdot F$ on $[\mu, \mu+\varphi(\omega, \mu)]$ such that $\mathfrak{S}(z)=\left[\mathfrak{S}_{*}(z), \mathfrak{S}^{*}(z)\right]$ for all $z \in[\mu, \mu+\varphi(\omega, \mu)]$. If $\varphi$ satisfies condition $C$ and $\mathfrak{S} \in L\left([\mu, \mu+\varphi(\omega, \mu)], \mathcal{K}_{C}^{+}\right)$, then:

$$
\begin{aligned}
\frac{1}{\alpha \chi\left(\frac{1}{2}\right)} \mathfrak{S}\left(\frac{2 \mu+\varphi(\omega, \mu)}{2}\right) & \leq_{p} \frac{\Gamma(\alpha)}{(\varphi(\omega, \mu))^{\alpha}}\left[\mathcal{I}_{\mu^{+}}^{\alpha} \mathfrak{S}(\mu+\varphi(\omega, \mu))+\mathcal{I}_{\mu+\varphi(\omega, \mu)^{-}} \mathfrak{S}(\mu)\right] \\
& \leq_{p}(\mathfrak{S}(\mu)+\mathfrak{S}(\mu+\varphi(\omega, \mu))) \int_{0}^{1} \varsigma^{\alpha-1}[\chi(\varsigma)-\chi(1-\varsigma)] d \varsigma \\
& \leq_{p}(\mathfrak{S}(\mu)+\mathfrak{S}(\omega)) \int_{0}^{1} \varsigma^{\alpha-1}[\chi(\varsigma)-\chi(1-\varsigma)] d \varsigma
\end{aligned}
$$

If $\mathfrak{S}(z)$ is pre-incave $I-V \cdot F$, then:

$$
\begin{aligned}
\frac{1}{\alpha \chi\left(\frac{1}{2}\right)} \mathfrak{S}\left(\frac{2 \mu+\varphi(\omega, \mu)}{2}\right) & \geq_{p} \frac{\Gamma(\alpha)}{(\varphi(\omega, \mu))^{\alpha}}\left[\mathcal{I}_{\mu^{+}}^{\alpha} \mathfrak{S}(\mu+\varphi(\omega, \mu))+\mathcal{I}_{\mu+\varphi(\omega, \mu)^{-}}^{\alpha} \mathfrak{S}(\mu)\right] \\
& \geq_{p}(\mathfrak{S}(\mu)+\mathfrak{S}(\mu+\varphi(\omega, \mu))) \int_{0}^{1} \varsigma^{\alpha-1}[\chi(\varsigma)-\chi(1-\varsigma)] d \varsigma \\
& \geq_{p}(\mathfrak{S}(\mu)+\mathfrak{S}(\omega)) \int_{0}^{1} \varsigma^{\alpha-1}[\chi(\varsigma)-\chi(1-\varsigma)] d \varsigma
\end{aligned}
$$

Proof. Let $\mathfrak{S}:[\mu, \mu+\varphi(\omega, \mu)] \rightarrow \mathcal{K}_{C}^{+}$be a LR--pre-invex $I-V \cdot F$. If condition $\mathrm{C}$ holds then, by hypothesis, we have:

$$
\frac{1}{\chi\left(\frac{1}{2}\right)} \mathfrak{S}\left(\frac{2 \mu+\varphi(\omega, \mu)}{2}\right) \leq_{p} \mathfrak{S}(\mu+(1-\varsigma) \varphi(\omega, \mu))+\mathfrak{S}(\mu+\varsigma \varphi(\omega, \mu)) .
$$

Therefore, we have:

$$
\begin{gathered}
\frac{1}{\chi\left(\frac{1}{2}\right)} \mathfrak{S}_{*}\left(\frac{2 \mu+\varphi(\omega, \mu)}{2}\right) \leq \mathfrak{S}_{*}(\mu+(1-\varsigma) \varphi(\omega, \mu))+\mathfrak{S}_{*}(\mu+\varsigma \varphi(\omega, \mu)), \\
\frac{1}{\chi\left(\frac{1}{2}\right)} \mathfrak{S}^{*}\left(\frac{2 \mu+\varphi(\omega, \mu)}{2}\right) \leq \mathfrak{S}^{*}(\mu+(1-\varsigma) \varphi(\omega, \mu))+\mathfrak{S}^{*}(\mu+\varsigma \varphi(\omega, \mu)) .
\end{gathered}
$$

Multiplying both sides by $\varsigma^{\alpha-1}$ and integrating the obtained result with respect to $\varsigma$ over $(0,1)$, we have

$$
\begin{aligned}
& \frac{1}{\chi\left(\frac{1}{2}\right)} \int_{0}^{1} \varsigma^{\alpha-1} \mathfrak{S}_{*}\left(\frac{2 \mu+\varphi(\omega, \mu)}{2}\right) d \varsigma \leq \int_{0}^{1} \varsigma^{\alpha-1} \mathfrak{S}_{*}(\mu+(1-\varsigma) \varphi(\omega, \mu)) d \varsigma+\int_{0}^{1} \varsigma^{\alpha-1} \mathfrak{S}_{*}(\mu+\varsigma \varphi(\omega, \mu)) d \varsigma, \\
& \frac{1}{\chi\left(\frac{1}{2}\right)} \int_{0}^{1} \varsigma^{\alpha-1} \mathfrak{S}^{*}\left(\frac{2 \mu+\varphi(\omega, \mu)}{2}\right) d \varsigma \leq \int_{0}^{1} \varsigma^{\alpha-1} \mathfrak{S}^{*}(\mu+(1-\varsigma) \varphi(\omega, \mu)) d \varsigma+\int_{0}^{1} \varsigma^{\alpha-1} \mathfrak{S}^{*}(\mu+\varsigma \varphi(\omega, \mu)) d \varsigma .
\end{aligned}
$$


Let $x=\mu+(1-\varsigma) \varphi(\omega, \mu)$ and $x=\mu+\varsigma \varphi(\omega, \mu)$. Then, we have:

$$
\begin{aligned}
\frac{1}{\alpha \chi\left(\frac{1}{2}\right)} \mathfrak{S}_{*}\left(\frac{2 \mu+\varphi(\omega, \mu)}{2}\right) & \leq \frac{1}{(\varphi(\omega, \mu))^{\alpha}} \int_{\mu}^{\mu+\varphi(\omega, \mu)}(\mu+\varphi(\omega, \mu)-x)^{\alpha-1} \mathfrak{S}_{*}(x) d x \\
& +\frac{1}{(\varphi(\omega, \mu))^{\alpha}} \int_{\mu}^{\mu+\varphi(\omega, \mu)}(z-\mu)^{\alpha-1} \mathfrak{S}_{*}(z) d z \\
\frac{1}{\alpha \chi\left(\frac{1}{2}\right)} \mathfrak{S}^{*}\left(\frac{2 \mu+\varphi(\omega, \mu)}{2}\right) & \leq \frac{1}{(\varphi(\omega, \mu))^{\alpha}} \int_{\mu}^{\mu+\varphi(\omega, \mu)}(\mu+\varphi(\omega, \mu)-x)^{\alpha-1} \mathfrak{S}^{*}(x) d x \\
& +\frac{1}{(\varphi(\omega, \mu))^{\alpha}} \int_{\mu}^{\mu+\varphi(\omega, \mu)}(z-\mu)^{\alpha-1} \mathfrak{S}^{*}(z) d z, \\
& \leq \frac{\Gamma(\alpha)}{(\varphi(\omega, \mu))^{\alpha}}\left[\mathcal{I}_{\mu^{+}}^{\alpha} \mathfrak{S}_{*}(\mu+\varphi(\omega, \mu))+\mathcal{I}_{\mu+\varphi(\omega, \mu)^{-}}^{\alpha} \mathfrak{S}_{*}(\mu)\right] \\
& \leq \frac{\Gamma(\alpha)}{(\varphi(\omega, \mu))^{\alpha}}\left[\mathcal{I}_{\mu^{+}}^{\alpha} \mathfrak{S}^{*}(\mu+\varphi(\omega, \mu))+\mathcal{I}_{\mu+\varphi(\omega, \mu)^{-}} \mathfrak{S}^{*}(\mu)\right],
\end{aligned}
$$

that is:

$$
\begin{aligned}
& \frac{1}{\alpha \chi\left(\frac{1}{2}\right)}\left[\mathfrak{S}_{*}\left(\frac{2 \mu+\varphi(\omega, \mu)}{2}\right), \mathfrak{S}^{*}\left(\frac{2 \mu+\varphi(\omega, \mu)}{2}\right)\right] \\
& \leq_{p} \frac{\Gamma(\alpha)}{(\varphi(\omega, \mu))^{\alpha}}\left[\mathcal{I}_{\mu^{+}}^{\alpha} \mathfrak{S}_{*}(\mu+\varphi(\omega, \mu))+\mathcal{I}_{\mu+\varphi(\omega, \mu)^{-}}^{\alpha} \mathfrak{S}_{*}(\mu), \mathcal{I}_{\mu^{+}}^{\alpha} \mathfrak{S}^{*}(\mu+\varphi(\omega, \mu))+\mathcal{I}_{\mu+\varphi(\omega, \mu)^{-}}^{\alpha} \mathfrak{S}^{*}(\mu)\right]
\end{aligned}
$$

thus,

$$
\frac{1}{\alpha \chi\left(\frac{1}{2}\right)} \mathfrak{S}\left(\frac{2 \mu+\varphi(\omega, \mu)}{2}\right) \leq_{p} \frac{\Gamma(\alpha)}{(\varphi(\omega, \mu))^{\alpha}}\left[\mathcal{I}_{\mu^{+}}^{\alpha} \mathfrak{S}(\mu+\varphi(\omega, \mu))+\mathcal{I}_{\mu+\varphi(\omega, \mu)^{-}}^{\alpha} \mathfrak{S}(\mu)\right] .
$$

In a similar way as above, we have:

$$
\begin{gathered}
\frac{\Gamma(\alpha)}{(\varphi(\omega, \mu))^{\alpha}}\left[\mathcal{I}_{\mu^{+}}^{\alpha} \mathfrak{S}(\mu+\varphi(\omega, \mu))+\mathcal{I}_{\mu+\varphi(\omega, \mu)^{-}}^{\alpha} \mathfrak{S}(\mu)\right] \\
\leq_{p}[\mathfrak{S}(\mu)+\mathfrak{S}(\mu+\varphi(\omega, \mu))] \int_{0}^{1} \varsigma^{\alpha-1}[\chi(\varsigma)-\chi(1-\varsigma)] d \varsigma .
\end{gathered}
$$

Combining (14) and (15), we have:

$$
\begin{aligned}
\frac{1}{\alpha \chi\left(\frac{1}{2}\right)} \mathfrak{S}\left(\frac{2 \mu+\varphi(\omega, \mu)}{2}\right) & \leq_{p} \frac{\Gamma(\alpha)}{(\varphi(\omega, \mu))^{\alpha}}\left[\mathcal{I}_{\mu^{+}}^{\alpha} \mathfrak{S}(\mu+\varphi(\omega, \mu))+\mathcal{I}_{\mu+\varphi(\omega, \mu)^{\alpha}} \mathfrak{S}(\mu)\right] \\
& \leq_{p}[\mathfrak{S}(\mu)+\mathfrak{S}(\mu+\varphi(\omega, \mu))] \int_{0}^{1} \varsigma^{\alpha-1}[\chi(\varsigma)-\chi(1-\varsigma)] d \varsigma \\
& \leq_{p}[\mathfrak{S}(\mu)+\mathfrak{S}(\omega)] \int_{0}^{1} \varsigma^{\alpha-1}[\chi(\varsigma)-\chi(1-\varsigma)] d \varsigma
\end{aligned}
$$

hence, the required result.

Remark 4. From Theorem 3 we clearly see that:

If $\varphi(\omega, \mu)=\omega-\mu$, then from Theorem 3 , we get the following new result in fractional calculus, see [42].

$$
\mathfrak{Q}\left(\frac{\mu+\omega}{2}\right) \leq_{p} \frac{\Gamma(\alpha+1)}{2(\omega-\mu)^{\alpha}}\left[\mathcal{I}_{\mu^{+}}^{\alpha} \mathfrak{Q}(\omega)+\mathcal{I}_{\omega^{-}}^{\alpha} \mathfrak{Q}(\mu)\right] \leq_{p} \frac{\mathfrak{Q}(\mu)+\mathfrak{Q}(\omega)}{2}
$$

If $\alpha=1$, then from Theorem 3, we obtain the following results for $L R$--pre-invex $I-V \cdot F$, which are also new ones:

$$
\begin{gathered}
\frac{1}{2 \chi\left(\frac{1}{2}\right)} \mathfrak{S}\left(\frac{2 \mu+\varphi(\omega, \mu)}{2}\right) \leq_{p} \frac{1}{\varphi(\omega, \mu)}(F R) \int_{\mu}^{\mu+\varphi(\omega, \mu)} \mathfrak{S}(z) d z \\
\leq_{p}[\mathfrak{S}(\mu)+\mathfrak{S}(\mu+\varphi(\omega, \mu))] \int_{0}^{1} \chi(\varsigma) d \varsigma .
\end{gathered}
$$

If $\chi(\varsigma)=\varsigma$, then Theorem 3 reduces to the result for LR-pre-invex I-V.F, see [41]:

$\mathfrak{S}\left(\frac{2 \mu+\varphi(\omega, \mu)}{2}\right) \leq_{p} \frac{\Gamma(\alpha+1)}{2(\varphi(\omega, \mu))^{\alpha}}\left[\mathcal{I}_{\mu^{+}}^{\alpha} \mathfrak{S}(\mu+\varphi(\omega, \mu))+\mathcal{I}_{\mu+\varphi(\omega, \mu)^{-}}^{\alpha} \mathfrak{S}(\mu)\right] \leq_{p} \frac{\mathfrak{S}(\mu)+\mathfrak{S}(\mu+\varphi(\omega, \mu))}{2}$ 
Let $\alpha=1$ and $\chi(\varsigma)=\varsigma$. Then, Theorem 3 reduces to the result for LR-pre-invex-I-V.F, see [41]:

$$
\mathfrak{S}\left(\frac{2 \mu+\varphi(\omega, \mu)}{2}\right) \leq_{p} \frac{1}{\varphi(\omega, \mu)}(F R) \int_{\mu}^{\mu+\varphi(\omega, \mu)} \mathfrak{S}(z) d z \leq_{p} \frac{\mathfrak{S}(\mu)+\mathfrak{S}(\omega)}{2}
$$

Example 2. $\alpha=\frac{1}{2}, \chi(\varsigma)=\varsigma$, for all $\varsigma \in[0,1]$ and the $I-V \cdot F \mathfrak{S}:[\mu, \mu+\varphi(\omega, \mu)]=[2,2+\varphi(3,2)] \rightarrow \mathcal{K}_{C^{\prime}}^{+}$ defined by $\mathfrak{S}(z)=[1,2]\left(2-z^{\frac{1}{2}}\right)$. Since left and right end-point functions $\mathfrak{S}_{*}(z)=\left(2-z^{\frac{1}{2}}\right)$, $\mathfrak{S}^{*}(z)=2\left(2-z^{\frac{1}{2}}\right)$, are $L R-\chi$-pre-invex functions, then $\mathfrak{S}(z)$ is $L R$ - $\chi$-pre-invex $I-V \cdot F$. We clearly see that $\mathfrak{S} \in L\left([\mu, \mu+\varphi(\omega, \mu)], \mathcal{K}_{C}^{+}\right)$and:

$$
\begin{gathered}
\frac{1}{\alpha \chi\left(\frac{1}{2}\right)} \mathfrak{S}_{*}\left(\frac{2 \mu+\varphi(\omega, \mu)}{2}\right)=\mathfrak{S}_{*}\left(\frac{5}{2}\right)=\frac{4-\sqrt{10}}{8} \\
\frac{1}{\alpha \chi\left(\frac{1}{2}\right)} \mathfrak{S}^{*}\left(\frac{2 \mu+\varphi(\omega, \mu)}{2}\right)=\mathfrak{S}^{*}\left(\frac{5}{2}\right)=\frac{4-\sqrt{10}}{4}, \\
\frac{\mathfrak{S}_{*}(\mu)+\mathfrak{S}_{*}(\mu+\varphi(\omega, \mu))}{2} \int_{0}^{1} \varsigma^{\alpha-1}[\chi(\varsigma)-\chi(1-\varsigma)] d \varsigma=(4-\sqrt{2}-\sqrt{3}) \\
\frac{\mathfrak{S}^{*}(\mu)+\mathfrak{S}^{*}(\mu+\varphi(\omega, \mu))}{2} \int_{0}^{1} \varsigma^{\alpha-1}[\chi(\varsigma)-\chi(1-\varsigma)] d \varsigma=2(4-\sqrt{2}-\sqrt{3}) .
\end{gathered}
$$

Note that:

$$
\begin{gathered}
\frac{\Gamma(\alpha)}{(\varphi(\omega, \mu))^{\alpha}}\left[\mathcal{I}_{\mu^{+}}^{\alpha} \mathfrak{S}_{*}(\mu+\varphi(\omega, \mu))+\mathcal{I}_{\mu+\varphi(\omega, \mu)^{-}}^{\alpha} \mathfrak{S}_{*}(\mu)\right] \\
=\frac{\Gamma\left(\frac{1}{2}\right)}{2} \frac{1}{\sqrt{\pi}} \int_{2}^{3}(3-z)^{\frac{-1}{2}} \cdot\left(2-z^{\frac{1}{2}}\right) d z \\
+\frac{\Gamma\left(\frac{1}{2}\right)}{2} \frac{1}{\sqrt{\pi}} \int_{2}^{3}(z-2)^{\frac{-1}{2}} \cdot\left(2-z^{\frac{1}{2}}\right) d z \\
=\frac{1}{2}\left[\frac{7393}{10,000}+\frac{9501}{10,000}\right] \\
=\frac{8447}{20,000} . \\
\frac{\Gamma(\alpha)}{(\varphi(\omega, \mu))^{\alpha}}\left[\mathcal{I}_{\mu^{+}}^{\alpha} \mathfrak{S}^{*}(\mu+\varphi(\omega, \mu))+\mathcal{I}_{\mu+\varphi(\omega, \mu)^{-}}^{\alpha} \mathfrak{S}^{*}(\mu)\right] \\
\frac{\Gamma(\alpha)}{(\varphi(\omega, \mu))^{\alpha}}\left[\mathcal{I}_{\mu^{+}}^{\alpha} \mathfrak{S}^{*}(\mu+\varphi(\omega, \mu))+\mathcal{I}_{\mu+\varphi(\omega, \mu)^{\alpha}}^{\alpha} \mathfrak{S}^{*}(\mu)\right] \\
+\frac{\Gamma\left(\frac{1}{2}\right)}{2} \frac{1}{\sqrt{\pi}} \int_{2}^{3}(z-2)^{\frac{-1}{2}} \cdot 2\left(2-z^{\frac{1}{2}}\right) d z \\
=\left[\frac{7393}{10,000}+\frac{9501}{10,000}\right] \\
=\frac{8447}{10,000} .
\end{gathered}
$$

Therefore:

$$
\left[\frac{4-\sqrt{10}}{8}, \frac{4-\sqrt{10}}{4}\right] \leq_{p}\left[\frac{8447}{20,000}, \frac{8447}{10,000}\right] \leq_{p}[(4-\sqrt{2}-\sqrt{3}), 2(4-\sqrt{2}-\sqrt{3})]
$$

and Theorem 3 is verified.

From Theorems 4 and 5, we obtain some interval fractional integral inequalities related to interval fractional $H-H$ inequalities. 
Theorem 4. Let $\mathfrak{S}, \mathcal{H}:[\mu, \mu+\varphi(\omega, \mu)] \rightarrow \mathcal{K}_{C}^{+}$be $L R-\chi_{1}$-pre-invex and $L R$ - $\chi_{2}$-pre-invex I-V.Fs on $[\mu, \mu+\varphi(\omega, \mu)]$, respectively, such that $\mathfrak{S}(z)=\left[\mathfrak{S}_{*}(z), \mathfrak{S}^{*}(z)\right]$ and $\mathcal{H}(z)=\left[\mathcal{H}_{*}(z), \mathcal{H}^{*}(z)\right]$ for all $z \in[\mu, \mu+\varphi(\omega, \mu)]$. If $\varphi$ satisfies condition $C$ and $\mathfrak{S} \times \mathcal{H} \in L\left([\mu, \mu+\varphi(\omega, \mu)], \mathcal{K}_{C}^{+}\right)$, then:

$$
\begin{aligned}
& \frac{\Gamma(\alpha)}{(\varphi(\omega, \mu))^{\alpha}}\left[\mathcal{I}_{\mu^{+}}^{\alpha} \mathfrak{S}(\mu+\varphi(\omega, \mu)) \times \mathcal{H}(\mu+\varphi(\omega, \mu))+\mathcal{I}_{\mu+\varphi(\omega, \mu)^{-}} \mathfrak{S}(\mu) \times \mathcal{H}(\mu)\right] \\
& \quad \leq_{p} \xi(\mu, \mu+\varphi(\omega, \mu)) \int_{0}^{1} \varsigma^{\alpha-1}\left[\chi_{1}(\varsigma) \chi_{2}(\varsigma)+\chi_{1}(1-\varsigma) \chi_{2}(1-\varsigma)\right] d \varsigma \\
& \quad+\partial(\mu, \mu+\varphi(\omega, \mu)) \int_{0}^{1} \varsigma^{\alpha-1}\left[\chi_{1}(\varsigma) \chi_{2}(1-\varsigma)+\chi_{1}(1-\varsigma) \chi_{2}(\varsigma)\right] d \varsigma .
\end{aligned}
$$

where $\xi(\mu, \mu+\varphi(\omega, \mu))=\mathfrak{S}(\mu) \times \mathcal{H}(\mu)+\mathfrak{S}(\mu+\varphi(\omega, \mu)) \times \mathcal{H}(\mu+\varphi(\omega, \mu))$, $\partial(\mu, \mu+\varphi(\omega, \mu))=\mathfrak{S}(\mu) \times \mathcal{H}(\mu+\varphi(\omega, \mu))+\mathfrak{S}(\mu+\varphi(\omega, \mu)) \times \mathcal{H}(\mu)$, and $\xi(\mu, \mu+\varphi(\omega, \mu))=\left[\xi_{*}((\mu, \mu+\varphi(\omega, \mu))), \xi^{*}((\mu, \mu+\varphi(\omega, \mu)))\right] \quad$ and $\partial(\mu, \mu+\varphi(\omega, \mu))=\left[\partial_{*}(\mu, \mu+\varphi(\omega, \mu)), \partial^{*}(\mu, \mu+\varphi(\omega, \mu))\right]$.

Proof. Since $\mathfrak{S}, \mathcal{H}$ both are LR- $\chi_{1}$-pre-invex and LR- $\chi_{2}$-pre-invex $I-V \cdot F$ then, we have:

$$
\begin{array}{r}
\mathfrak{S}_{*}(\mu+(1-\varsigma) \varphi(\omega, \mu))=\mathfrak{S}_{*}(\mu+\varphi(\omega, \mu)+\varsigma \varphi(\mu, \mu+\varphi(\omega, \mu))) \\
\leq \chi_{1}(\varsigma) \mathfrak{S}_{*}(\mu)+\chi_{1}(1-\varsigma) \mathfrak{S}_{*}(\mu+\varphi(\omega, \mu)) \\
\mathfrak{S}^{*}(\mu+(1-\varsigma) \varphi(\omega, \mu))=\mathfrak{S}^{*}(\mu+\varphi(\omega, \mu)+\varsigma \varphi(\mu, \mu+\varphi(\omega, \mu))) \\
\leq \chi_{1}(\varsigma) \mathfrak{S}^{*}(\mu)+\chi_{1}(1-\varsigma) \mathfrak{S}^{*}(\mu+\varphi(\omega, \mu)) .
\end{array}
$$

and:

$$
\begin{aligned}
\mathcal{H}_{*}(\mu+(1-\varsigma) \varphi(\omega, \mu))= & \mathcal{H}_{*}(\mu+(1-\varsigma) \varphi(\omega, \mu)) \\
& \leq \chi_{2}(\varsigma) \mathcal{H}_{*}(\mu)+\chi_{2}(1-\varsigma) \mathcal{H}_{*}(\mu+\varphi(\omega, \mu)) \\
\mathcal{H}^{*}(\mu+(1-\varsigma) \varphi(\omega, \mu))= & \mathcal{H}^{*}(\mu+(1-\varsigma) \varphi(\omega, \mu)) \\
& \leq \chi_{2}(\varsigma) \mathcal{H}^{*}(\mu)+\chi_{2}(1-\varsigma) \mathcal{H}^{*}(\mu+\varphi(\omega, \mu)) .
\end{aligned}
$$

From the definition of LR--pre-invex $I-V \cdot F$, we have:

$$
\begin{aligned}
\mathfrak{S}_{*}(\mu & +(1-\varsigma) \varphi(\omega, \mu)) \times \mathcal{H}_{*}(\mu+(1-\varsigma) \varphi(\omega, \mu)) \\
& \leq \chi_{1}(\varsigma) \chi_{2}(\varsigma) \mathfrak{S}_{*}(\mu) \times \mathcal{H}_{*}(\mu)+\chi_{1}(1-\varsigma) \chi_{2}(1-\varsigma) \mathfrak{S}_{*}(\mu+\varphi(\omega, \mu)) \times \mathcal{H}_{*}(\mu+\varphi(\omega, \mu)) \\
& +\chi_{1}(\varsigma) \chi_{2}(1-\varsigma) \mathfrak{S}_{*}(\mu) \times \mathcal{H}_{*}(\mu+\varphi(\omega, \mu))+\chi_{1}(1-\varsigma) \chi_{2}(\varsigma) \mathfrak{S}_{*}(\mu+\varphi(\omega, \mu)) \times \mathcal{H}_{*}(\mu) \\
\mathfrak{S}^{*}(\mu & +(1-\varsigma) \varphi(\omega, \mu)) \times \mathcal{H}^{*}(\mu+(1-\varsigma) \varphi(\omega, \mu)) \\
& \leq \chi_{1}(\varsigma) \chi_{2}(\varsigma) \mathfrak{S}^{*}(\mu) \times \mathcal{H}^{*}(\mu)+\chi_{1}(1-\varsigma) \chi_{2}(1-\varsigma) \mathfrak{S}^{*}(\mu+\varphi(\omega, \mu)) \times \mathcal{H}^{*}(\mu+\varphi(\omega, \mu)) \\
& +\chi_{1}(\varsigma) \chi_{2}(1-\varsigma) \mathfrak{S}^{*}(\mu) \times \mathcal{H}^{*}(\mu+\varphi(\omega, \mu))+\chi_{1}(1-\varsigma) \chi_{2}(\varsigma) \mathfrak{S}^{*}(\mu+\varphi(\omega, \mu)) \times \mathcal{H}^{*}(\mu) .
\end{aligned}
$$

Analogously, we have:

$$
\begin{aligned}
& \mathfrak{S}_{*}(\mu+\varsigma \varphi(\omega, \mu)) \mathcal{H}_{*}(\mu+\varsigma \varphi(\omega, \mu)) \\
& \leq \chi_{1}(1-\varsigma) \chi_{2}(1-\varsigma) \mathfrak{S}_{*}(\mu) \times \mathcal{H}_{*}(\mu)+\chi_{1}(\varsigma) \chi_{2}(\varsigma) \mathfrak{S}_{*}(\mu+\varphi(\omega, \mu)) \times \mathcal{H}_{*}(\mu+\varphi(\omega, \mu)) \\
& +\chi_{1}(1-\varsigma) \chi_{2}(\varsigma) \mathfrak{S}_{*}(\mu) \times \mathcal{H}_{*}(\mu+\varphi(\omega, \mu))+\chi_{1}(\varsigma) \chi_{2}(1-\varsigma) \mathfrak{S}_{*}(\mu+\varphi(\omega, \mu)) \times \mathcal{H}_{*}(\mu) \\
& \mathfrak{S}^{*}(\mu+\varsigma \varphi(\omega, \mu)) \times \mathcal{H}^{*}(\mu+\varsigma \varphi(\omega, \mu)) \\
& \leq \chi_{1}(1-\varsigma) \chi_{2}(1-\varsigma) \mathfrak{S}^{*}(\mu) \times \mathcal{H}^{*}(\mu)+\chi_{1}(\varsigma) \chi_{2}(\varsigma) \mathfrak{S}^{*}(\mu+\varphi(\omega, \mu)) \times \mathcal{H}^{*}(\mu+\varphi(\omega, \mu)) \\
& +\chi_{1}(1-\varsigma) \chi_{2}(\varsigma) \mathfrak{S}^{*}(\mu) \times \mathcal{H}^{*}(\mu+\varphi(\omega, \mu))+\chi_{1}(\varsigma) \chi_{2}(1-\varsigma) \mathfrak{S}^{*}(\mu+\varphi(\omega, \mu)) \times \mathcal{H}^{*}(\mu) \text {. }
\end{aligned}
$$

Adding (21) and (22), we have: 


$$
\begin{gathered}
\mathfrak{S}_{*}(\mu+(1-\varsigma) \varphi(\omega, \mu)) \times \mathcal{H}_{*}(\mu+(1-\varsigma) \varphi(\omega, \mu))+\mathfrak{S}_{*}(\mu+\varsigma \varphi(\omega, \mu)) \times \mathcal{H}_{*}(\mu+\varsigma \varphi(\omega, \mu)) \\
\leq\left[\begin{array}{c}
\chi_{1}(\varsigma) \chi_{2}(\varsigma) \\
+\chi_{1}(1-\varsigma) \chi_{2}(1-\varsigma)
\end{array}\right]\left[\mathfrak{S}_{*}(\mu) \times \mathcal{H}_{*}(\mu)+\mathfrak{S}_{*}(\mu+\varphi(\omega, \mu)) \times \mathcal{H}_{*}(\mu+\varphi(\omega, \mu))\right] \\
+\left[\begin{array}{c}
\chi_{1}(\varsigma) \chi_{2}(1-\varsigma) \\
+\chi_{1}(1-\varsigma) \chi_{2}(\varsigma)
\end{array}\right]\left[\mathfrak{S}_{*}(\mu+\varphi(\omega, \mu)) \times \mathcal{H}_{*}(\mu)+\mathfrak{S}_{*}(\mu) \times \mathcal{H}_{*}(\mu+\varphi(\omega, \mu))\right] \\
\mathfrak{S}^{*}(\mu+(1-\varsigma) \varphi(\omega, \mu)) \times \mathcal{H}^{*}(\mu+(1-\varsigma) \varphi(\omega, \mu))+\mathfrak{S}^{*}(\mu+\varsigma \varphi(\omega, \mu)) \times \mathcal{H}^{*}(\mu+\varsigma \varphi(\omega, \mu)) \\
\leq\left[\begin{array}{c}
\chi_{1}(\varsigma) \chi_{2}(\varsigma) \\
+\chi_{1}(1-\varsigma) \chi_{2}(1-\varsigma)
\end{array}\right]\left[\mathfrak{S}^{*}(\mu) \times \mathcal{H}^{*}(\mu)+\mathfrak{S}^{*}(\mu+\varphi(\omega, \mu)) \times \mathcal{H}^{*}(\mu+\varphi(\omega, \mu))\right] \\
+\left[\begin{array}{c}
\chi_{1}(\varsigma) \chi_{2}(1-\varsigma) \\
+\chi_{1}(1-\varsigma) \chi_{2}(\varsigma)
\end{array}\right]\left[\mathfrak{S}^{*}(\mu+\varphi(\omega, \mu)) \times \mathcal{H}^{*}(\mu)+\mathfrak{S}^{*}(\mu) \times \mathcal{H}^{*}(\mu+\varphi(\omega, \mu))\right] .
\end{gathered}
$$

Taking multiplication of (23) with $\varsigma^{\alpha-1}$ and integrating the obtained result with respect to $\varsigma$ over $(0,1)$, we have:

$$
\begin{aligned}
& \int_{0}^{1} \varsigma^{\alpha-1} \mathfrak{S}_{*}(\mu+(1-\varsigma) \varphi(\omega, \mu)) \times \mathcal{H}_{*}(\mu+(1-\varsigma) \varphi(\omega, \mu)) \\
&+\varsigma^{\alpha-1} \mathfrak{S}_{*}(\mu+\varsigma \varphi(\omega, \mu)) \times \mathcal{H}_{*}(\mu+\varsigma \varphi(\omega, \mu)) d \varsigma \\
& \leq \xi_{*}((\mu, \mu+\varphi(\omega, \mu))) \int_{0}^{1} \varsigma^{\alpha-1}\left[\chi_{1}(\varsigma) \chi_{2}(\varsigma)+\chi_{1}(1-\varsigma) \chi_{2}(1-\varsigma)\right] d \varsigma \\
&+\partial_{*}((\mu, \mu+\varphi(\omega, \mu))) \int_{0}^{1} \varsigma^{\alpha-1}\left[\chi_{1}(\varsigma) \chi_{2}(1-\varsigma)+\chi_{1}(1-\varsigma) \chi_{2}(\varsigma)\right] d \varsigma \\
& \int_{0}^{1} \varsigma^{\alpha-1} \mathfrak{S}^{*}(\mu+(1-\varsigma) \varphi(\omega, \mu)) \times \mathcal{H}^{*}(\mu+(1-\varsigma) \varphi(\omega, \mu)) \\
& \quad+\varsigma^{\alpha-1} \mathfrak{S}^{*}(\mu+\varsigma \varphi(\omega, \mu)) \times \mathcal{H}^{*}(\mu+\varsigma \varphi(\omega, \mu)) d \varsigma \\
& \leq \zeta^{*}((\mu, \mu+\varphi(\omega, \mu))) \int_{0}^{1} \varsigma^{\alpha-1}\left[\chi_{1}(\varsigma) \chi_{2}(\varsigma)+\chi_{1}(1-\varsigma) \chi_{2}(1-\varsigma)\right] d \varsigma \\
&+\partial^{*}((\mu, \mu+\varphi(\omega, \mu))) \int_{0}^{1} \varsigma^{\alpha-1}\left[\chi_{1}(\varsigma) \chi_{2}(1-\varsigma)+\chi_{1}(1-\varsigma) \chi_{2}(\varsigma)\right] d \varsigma .
\end{aligned}
$$

It follows that:

$$
\begin{aligned}
\frac{\Gamma(\alpha)}{(\varphi(\omega, \mu))^{\alpha}} & {\left[\mathcal{I}_{\mu^{+}}^{\alpha} \mathfrak{S}_{*}(\mu+\varphi(\omega, \mu)) \times \mathcal{H}_{*}(\mu+\varphi(\omega, \mu))+\mathcal{I}_{\mu+\varphi(\omega, \mu)^{-}}^{\alpha} \mathfrak{S}_{*}(\mu) \times \mathcal{H}_{*}(\mu)\right] } \\
\leq & \xi_{*}((\mu, \mu+\varphi(\omega, \mu))) \int_{0}^{1} \varsigma^{\alpha-1}\left[\chi_{1}(\varsigma) \chi_{2}(\varsigma)+\chi_{1}(1-\varsigma) \chi_{2}(1-\varsigma)\right] d \varsigma \\
& +\partial_{*}((\mu, \mu+\varphi(\omega, \mu))) \int_{0}^{1} \varsigma^{\alpha-1}\left[\chi_{1}(\varsigma) \chi_{2}(1-\varsigma)+\chi_{1}(1-\varsigma) \chi_{2}(\varsigma)\right] d \varsigma \\
\frac{\Gamma(\alpha)}{(\varphi(\omega, \mu))^{\alpha}} & {\left[\mathcal{I}_{\mu^{+}}^{\alpha} \mathfrak{S}^{*}(\mu+\varphi(\omega, \mu)) \times \mathcal{H}^{*}(\mu+\varphi(\omega, \mu))+\mathcal{I}_{\mu+\varphi(\omega, \mu)}^{\alpha} \mathfrak{S}^{*}(\mu) \times \mathcal{H}^{*}(\mu)\right] } \\
\leq & \xi^{*}((\mu, \mu+\varphi(\omega, \mu))) \int_{0}^{1} \varsigma^{\alpha-1}\left[\chi_{1}(\varsigma) \chi_{2}(\varsigma)+\chi_{1}(1-\varsigma) \chi_{2}(1-\varsigma)\right] d \varsigma \\
& \left.+\partial^{*}((\mu, \mu+] \varphi(\omega, \mu))\right) \int_{0}^{1} \varsigma^{\alpha-1}\left[\chi_{1}(\varsigma) \chi_{2}(1-\varsigma)+\chi_{1}(1-\varsigma) \chi_{2}(\varsigma)\right] d \varsigma .
\end{aligned}
$$

It results that:

\footnotetext{
$\frac{\Gamma(\alpha)}{(\varphi(\omega, \mu))^{\alpha}}\left[\mathcal{I}_{\mu^{+}}^{\alpha} \mathfrak{S}_{*}(\mu+\varphi(\omega, \mu)) \times \mathcal{H}_{*}(\mu+\varphi(\omega, \mu))+\mathcal{I}_{\mu+}^{\alpha}\right.$

$\left.\mathfrak{S}_{*}(\mu) \times \mathcal{H}_{*}(\mu), \quad \mathcal{I}_{\mu^{+}}^{\alpha} \mathfrak{S}^{*}(\mu+\varphi(\omega, \mu)) \times \mathcal{H}^{*}(\mu+\varphi(\omega, \mu))+\mathcal{I}_{\mu+\varphi(\omega, \mu)^{-}}^{\alpha} \mathfrak{S}^{*}(\mu) \times \mathcal{H}^{*}(\mu)\right]$ $\leq_{p}\left[\xi_{*}((\mu, \mu+\varphi(\omega, \mu))), \xi^{*}((\mu, \mu+\varphi(\omega, \mu)))\right] \int_{0}^{1} \varsigma^{\alpha-1}\left[\chi_{1}(\varsigma) \chi_{2}(\varsigma)+\chi_{1}(1-\varsigma) \chi_{2}(1-\varsigma)\right] d \varsigma$ $+\left[\partial_{*}((\mu, \mu+\varphi(\omega, \mu))), \partial^{*}((\mu, \mu+\varphi(\omega, \mu)))\right] \int_{0}^{1} \varsigma^{\alpha-1}\left[\chi_{1}(\varsigma) \chi_{2}(1-\varsigma)+\chi_{1}(1-\varsigma) \chi_{2}(\varsigma)\right] d \varsigma$
}

that is:

$$
\begin{gathered}
\frac{\Gamma(\alpha)}{(\varphi(\omega, \mu))^{\alpha}}\left[\mathcal{I}_{\mu^{+}}^{\alpha} \mathfrak{S}(\mu+\varphi(\omega, \mu)) \times \mathcal{H}(\mu+\varphi(\omega, \mu))+\mathcal{I}_{\mu+\varphi(\omega, \mu)^{\alpha}}^{\alpha} \mathfrak{S}(\mu) \times \mathcal{H}(\mu)\right] \\
\quad \leq_{p} \xi(\mu, \mu+\varphi(\omega, \mu)) \int_{0}^{1} \varsigma^{\alpha-1}\left[\chi_{1}(\varsigma) \chi_{2}(\varsigma)+\chi_{1}(1-\varsigma) \chi_{2}(1-\varsigma)\right] d \varsigma \\
\quad+\partial(\mu, \mu+\varphi(\omega, \mu)) \int_{0}^{1} \varsigma^{\alpha-1}\left[\chi_{1}(\varsigma) \chi_{2}(1-\varsigma)+\chi_{1}(1-\varsigma) \chi_{2}(\varsigma)\right] d \varsigma
\end{gathered}
$$

and the theorem has been established.

Theorem 5. Let $\mathfrak{S}, \mathcal{H}:[\mu, \mu+\varphi(\omega, \mu)] \rightarrow \mathcal{K}_{C}^{+}$be two LR- $\chi_{1}$-pre-invex and LR- $\chi_{2}$-pre-invex $I-V \cdot F S$, respectively, such that $\mathfrak{S}(z)=\left[\mathfrak{S}_{*}(z), \mathfrak{S}^{*}(z)\right]$ and $\mathcal{H}(z)=\left[\mathcal{H}_{*}(z), \mathcal{H}^{*}(z)\right]$ for all $z \in[\mu, \mu+\varphi(\omega, \mu)]$. If $\varphi$ satisfies condition $C$ and $\mathfrak{S} \times \mathcal{H} \in L\left([\mu, \mu+\varphi(\omega, \mu)], \mathcal{K}_{C}^{+}\right)$, then: 


$$
\begin{gathered}
\frac{1}{\alpha \chi_{1}\left(\frac{1}{2}\right) \chi_{2}\left(\frac{1}{2}\right)} \mathfrak{S}\left(\frac{2 \mu+\varphi(\omega, \mu)}{2}\right) \times \mathcal{H}\left(\frac{2 \mu+\varphi(\omega, \mu)}{2}\right) \\
\leq_{p} \frac{\Gamma(\alpha)}{(\varphi(\omega, \mu))^{\alpha}}\left[\mathcal{I}_{\mu^{+}}^{\alpha} \mathfrak{S}(\mu+\varphi(\omega, \mu)) \times \mathcal{H}(\omega)+\mathcal{I}_{\mu+\varphi(\omega, \mu)^{\alpha}}^{\alpha} \mathfrak{S}(\mu) \times \mathcal{H}(\mu)\right] \\
+\partial(\mu, \mu+\varphi(\omega, \mu)) \int_{0}^{1}\left[\varsigma^{\alpha-1}+(1-\varsigma)^{\alpha-1}\right] \chi_{1}(\varsigma) \chi_{2}(1-\varsigma) d \varsigma \\
+\xi(\mu, \mu+\varphi(\omega, \mu)) \int_{0}^{1}\left[\varsigma^{\alpha-1}+(1-\varsigma)^{\alpha-1}\right] \chi_{1}(1-\varsigma) \chi_{2}(1-\varsigma) d \varsigma,
\end{gathered}
$$

where $\xi(u, u+\varphi(v, u))=\mathfrak{S}(u) \times \mathcal{H}(u)+\mathfrak{S}(\mu+\varphi(\omega, \mu)) \times \mathcal{H}(\mu+\varphi(\omega, \mu))$, $\partial(\mu, \mu+\varphi(\omega, \mu))=\mathfrak{S}(\mu) \times \mathcal{H}(\mu+\varphi(\omega, \mu))+\mathfrak{S}(\mu+\varphi(\omega, \mu)) \times \mathcal{H}(\mu)$, and $\xi(\mu, \mu+\varphi(\omega, \mu)) \quad=\quad\left[\xi_{*}(\mu, \mu+\varphi(\omega, \mu)), \xi^{*}(\mu, \mu+\varphi(\omega, \mu))\right] \quad$ and $\partial(\mu, \mu+\varphi(\omega, \mu))=\left[\partial_{*}((\mu, \mu+\varphi(\omega, \mu))), \partial^{*}(\mu, \mu+\varphi(\omega, \mu))\right]$.

Proof. Consider $\mathfrak{S}, \mathcal{H}:[\mu, \mu+\varphi(\omega, \mu)] \rightarrow \mathcal{K}_{C}^{+}$. are LR- $\chi_{1}$-pre-invex and LR- $\chi_{2}$-pre-invex $I-V \cdot F s$. Then, by hypothesis, we have:

$$
\begin{aligned}
& \begin{array}{c}
\mathfrak{S}_{*}\left(\frac{2 \mu+\varphi(\omega, \mu)}{2}\right) \times \mathcal{H}_{*}\left(\frac{2 \mu+\varphi(\omega, \mu)}{2}\right) \\
\mathfrak{S}^{*}\left(\frac{2 \mu+\varphi(\omega, \mu)}{2}\right) \times \mathcal{H}^{*}\left(\frac{2 \mu+\varphi(\omega, \mu)}{2}\right)
\end{array} \\
& \leq \chi_{1}\left(\frac{1}{2}\right) \chi_{2}\left(\frac{1}{2}\right)\left[\begin{array}{c}
\mathfrak{S}_{*}(\mu+(1-\varsigma) \varphi(\omega, \mu)) \times \mathcal{H}_{*}(\mu+(1-\varsigma) \varphi(\omega, \mu)) \\
+\mathfrak{S}_{*}(\mu+(1-\varsigma) \varphi(\omega, \mu)) \times \mathcal{H}_{*}(\mu+\varsigma \varphi(\omega, \mu))
\end{array}\right] \\
& +\chi_{1}\left(\frac{1}{2}\right) \chi_{2}\left(\frac{1}{2}\right)\left[\begin{array}{c}
\mathfrak{S}_{*}(\mu+\varsigma \varphi(\omega, \mu)) \times \mathcal{H}_{*}(\mu+(1-\varsigma) \varphi(\omega, \mu)) \\
+\mathfrak{S}_{*}(\mu+\varsigma \varphi(\omega, \mu)) \times \mathcal{H}_{*}(\mu+\varsigma \varphi(\omega, \mu))
\end{array}\right] \\
& \leq \chi_{1}\left(\frac{1}{2}\right) \chi_{2}\left(\frac{1}{2}\right)\left[\begin{array}{c}
\mathfrak{S}^{*}(\mu+(1-\varsigma) \varphi(\omega, \mu)) \times \mathcal{H}^{*}(\mu+(1-\varsigma) \varphi(\omega, \mu)) \\
+\mathfrak{S}^{*}(\mu+(1-\varsigma) \varphi(\omega, \mu)) \times \mathcal{H}^{*}(\mu+\varsigma \varphi(\omega, \mu))
\end{array}\right] \\
& +\chi_{1}\left(\frac{1}{2}\right) \chi_{2}\left(\frac{1}{2}\right)\left[\begin{array}{c}
\mathfrak{S}^{*}(\mu+\varsigma \varphi(\omega, \mu)) \times \mathcal{H}^{*}(\mu+(1-\varsigma) \varphi(\omega, \mu)) \\
+\mathfrak{S}^{*}(\mu+\varsigma \varphi(\omega, \mu)) \times \mathcal{H}^{*}(\mu+\varsigma \varphi(\omega, \mu))
\end{array}\right], \\
& \leq \chi_{1}\left(\frac{1}{2}\right) \chi_{2}\left(\frac{1}{2}\right)\left[\begin{array}{c}
\mathfrak{S}_{*}(\mu+(1-\varsigma) \varphi(\omega, \mu)) \times \mathcal{H}_{*}(\mu+(1-\varsigma) \varphi(\omega, \mu)) \\
+\mathfrak{S}_{*}(\mu+\varsigma \varphi(\omega, \mu)) \times \mathcal{H}_{*}(\mu+\varsigma \varphi(\omega, \mu))
\end{array}\right] \\
& +\chi_{1}\left(\frac{1}{2}\right) \chi_{2}\left(\frac{1}{2}\right)\left[\begin{array}{c}
\left(\chi_{1}(\varsigma) \mathfrak{S}_{*}(\mu)+\chi_{1}(1-\varsigma) \mathfrak{S}_{*}(\mu+\varphi(\omega, \mu),)\right) \\
\times\left(\chi_{2}(1-\varsigma) \mathcal{H}_{*}(\mu)+\chi_{2}(\varsigma) \mathcal{H}_{*}(\mu+\varphi(\omega, \mu),)\right) \\
+\left(\chi_{1}(1-\varsigma) \mathfrak{S}_{*}(\mu)+\chi_{1}(\varsigma) \mathfrak{S}_{*}(\mu+\varphi(\omega, \mu),)\right) \\
\times\left(\chi_{2}(\varsigma) \mathcal{H}_{*}(\mu)+\chi_{2}(1-\varsigma) \mathcal{H}_{*}(\mu+\varphi(\omega, \mu),)\right)
\end{array}\right] \\
& \leq \chi_{1}\left(\frac{1}{2}\right) \chi_{2}\left(\frac{1}{2}\right)\left[\begin{array}{c}
\mathfrak{S}^{*}(\mu+(1-\varsigma) \varphi(\omega, \mu)) \times \mathcal{H}^{*}(\mu+(1-\varsigma) \varphi(\omega, \mu)) \\
+\mathfrak{S}^{*}(\mu+\varsigma \varphi(\omega, \mu)) \times \mathcal{H}^{*}(\mu+\varsigma \varphi(\omega, \mu))
\end{array}\right] \\
& +\chi_{1}\left(\frac{1}{2}\right) \chi_{2}\left(\frac{1}{2}\right)\left[\begin{array}{c}
\left(\chi_{1}(\varsigma) \mathfrak{S}^{*}(\mu)+\chi_{1}(1-\varsigma) \mathfrak{S}^{*}(\mu+\varphi(\omega, \mu),)\right) \\
\times\left(\chi_{2}(1-\varsigma) \mathcal{H}^{*}(\mu)+\chi_{2}(\varsigma) \mathcal{H}^{*}(\mu+\varphi(\omega, \mu),)\right) \\
+\left(\chi_{1}(1-\varsigma) \mathfrak{S}^{*}(\mu)+\chi_{1}(\varsigma) \mathfrak{S}^{*}(\mu+\varphi(\omega, \mu),)\right) \\
\times\left(\chi_{2}(\varsigma) \mathcal{H}^{*}(\mu)+\chi_{2}(1-\varsigma) \mathcal{H}^{*}(\mu+\varphi(\omega, \mu),)\right)
\end{array}\right] \\
& =\chi_{1}\left(\frac{1}{2}\right) \chi_{2}\left(\frac{1}{2}\right)\left[\begin{array}{c}
\mathfrak{S}_{*}(\mu+(1-\varsigma) \varphi(\omega, \mu)) \times \mathcal{H}_{*}(\mu+(1-\varsigma) \varphi(\omega, \mu)) \\
+\mathfrak{S}_{*}(\mu+\varsigma \varphi(\omega, \mu)) \times \mathcal{H}_{*}(\mu+\varsigma \varphi(\omega, \mu))
\end{array}\right] \\
& +\chi_{1}\left(\frac{1}{2}\right) \chi_{2}\left(\frac{1}{2}\right)\left[\begin{array}{c}
\left\{\chi_{1}(\varsigma) \chi_{2}(1-\varsigma)+\chi_{1}(1-\varsigma) \chi_{2}(\varsigma)\right\} \partial_{*}((\mu, \mu+\varphi(\omega, \mu))) \\
+\left\{\chi_{1}(\varsigma) \chi_{2}(\varsigma)+\chi_{1}(1-\varsigma) \chi_{2}(1-\varsigma)\right\} \xi_{*}((\mu, \mu+\varphi(\omega, \mu)))
\end{array}\right] \\
& =\chi_{1}\left(\frac{1}{2}\right) \chi_{2}\left(\frac{1}{2}\right)\left[\begin{array}{c}
\mathfrak{S}^{*}(\mu+(1-\varsigma) \varphi(\omega, \mu)) \times \mathcal{H}^{*}(\mu+(1-\varsigma) \varphi(\omega, \mu)) \\
+\mathfrak{S}^{*}(\mu+\varsigma \varphi(\omega, \mu)) \times \mathcal{H}^{*}(\mu+\varsigma \varphi(\omega, \mu))
\end{array}\right] \\
& +\chi_{1}\left(\frac{1}{2}\right) \chi_{2}\left(\frac{1}{2}\right)\left[\begin{array}{c}
\left\{\chi_{1}(\varsigma) \chi_{2}(1-\varsigma)+\chi_{1}(1-\varsigma) \chi_{2}(\varsigma)\right\} \partial^{*}(\mu, \mu+\varphi(\omega, \mu)) \\
+\left\{\chi_{1}(\varsigma) \chi_{2}(\varsigma)+\chi_{1}(1-\varsigma) \chi_{2}(1-\varsigma)\right\} \xi^{*}(\mu, \mu+\varphi(\omega, \mu))
\end{array}\right] .
\end{aligned}
$$


Taking multiplication of (25) with $\varsigma^{\alpha-1}$ and integrating over $(0,1)$, we get:

$$
\begin{aligned}
& \frac{1}{\alpha \chi_{1}\left(\frac{1}{2}\right) \chi_{2}\left(\frac{1}{2}\right)} \mathfrak{S}_{*}\left(\frac{2 \mu+\varphi(\omega, \mu)}{2}\right) \times \mathcal{H}_{*}\left(\frac{2 \mu+\varphi(\omega, \mu)}{2}\right) \\
& \leq \frac{\Gamma(\alpha)}{(\varphi(\omega, \mu))^{\alpha}}\left[\mathcal{I}_{\mu^{+}}^{\alpha} \mathfrak{S}_{*}(\mu+\varphi(\omega, \mu)) \times \mathcal{H}_{*}(\mu+\varphi(\omega, \mu))+\mathcal{I}_{\mu+\varphi(\omega, \mu)^{-}}^{\alpha} \mathfrak{S}_{*}(\mu) \times \mathcal{H}_{*}(\mu)\right] \\
& \quad+\partial_{*}(\mu, \mu+\varphi(\omega, \mu)) \int_{0}^{1}\left[\varsigma^{\alpha-1}+(1-\varsigma)^{\alpha-1}\right] \chi_{1}(\varsigma) \chi_{2}(1-\varsigma) d \varsigma b \\
& \quad+\xi_{*}(\mu, \mu+\varphi(\omega, \mu)) \int_{0}^{1}\left[\varsigma^{\alpha-1}+(1-\varsigma)^{\alpha-1}\right] \chi_{1}(1-\varsigma) \chi_{2}(1-\varsigma) d \varsigma \\
& \frac{1}{\alpha \chi_{1}\left(\frac{1}{2}\right) \chi_{2}\left(\frac{1}{2}\right)} \mathfrak{S}^{*}\left(\frac{2 \mu+\varphi(\omega, \mu)}{2}\right) \times \mathcal{H}^{*}\left(\frac{2 \mu+\varphi(\omega, \mu)}{2}\right) \\
& \leq \frac{\Gamma(\alpha)}{(\varphi(\omega, \mu))^{\alpha}}\left[\mathcal{I}_{\mu^{+}}^{\alpha} \mathfrak{S}^{*}(\mu+\varphi(\omega, \mu)) \times \mathcal{H}^{*}(\mu+\varphi(\omega, \mu))+\mathcal{I}_{\mu+\varphi(\omega, \mu)^{-}}^{\alpha} \mathfrak{S}^{*}(\mu) \times \mathcal{H}^{*}(\mu)\right] \\
& \quad+\partial^{*}(\mu, \mu+\varphi(\omega, \mu)) \int_{0}^{1}\left[\varsigma^{\alpha-1}+(1-\varsigma)^{\alpha-1}\right] \chi_{1}(\varsigma) \chi_{2}(1-\varsigma) d \varsigma \\
& \quad+\xi^{*}(\mu, \mu+\varphi(\omega, \mu)) \int_{0}^{1}\left[\varsigma^{\alpha-1}+(1-\varsigma)^{\alpha-1}\right] \chi_{1}(1-\varsigma) \chi_{2}(1-\varsigma) d \varsigma .
\end{aligned}
$$

It follows that:

$$
\begin{gathered}
\frac{1}{\alpha \chi_{1}\left(\frac{1}{2}\right) \chi_{2}\left(\frac{1}{2}\right)} \mathfrak{S}\left(\frac{2 \mu+\varphi(\omega, \mu)}{2}\right) \times \mathcal{H}\left(\frac{2 \mu+\varphi(\omega, \mu)}{2}\right) \\
\leq p \frac{\Gamma(\alpha)}{(\varphi(\omega, \mu))^{\alpha}}\left[\mathcal{I}_{\mu^{+}}^{\alpha} \mathfrak{S}(\mu+\varphi(\omega, \mu)) \times \mathcal{H}(\mu+\varphi(\omega, \mu))\right. \\
\left.+\mathcal{I}_{\mu+\varphi(\omega, \mu)^{-}} \mathfrak{S}(\mu) \times \mathcal{H}(\mu)\right] \\
+\partial(\mu, \mu+\varphi(\omega, \mu)) \int_{0}^{1}\left[\varsigma^{\alpha-1}+(1-\varsigma)^{\alpha-1}\right] \chi_{1}(\varsigma) \chi_{2}(1-\varsigma) d \varsigma \\
+\xi(\mu, \mu+\varphi(\omega, \mu)) \int_{0}^{1}\left[\varsigma^{\alpha-1}+(1-\varsigma)^{\alpha-1}\right] \chi_{1}(1-\varsigma) \chi_{2}(1-\varsigma) d \varsigma
\end{gathered}
$$

Hence, the required result.

Now, we present the successful reformative version of the generalized version of interval $H$ - $H$ inequality on invex set for LR- $\chi$-pre-invex $I-V \cdot F$ via interval Riemann-Liouville fractional integral.

Theorem 6. (Second fractional $H$-H Fejér inequality) Let $\mathfrak{S}:[\mu, \mu+\varphi(\omega, \mu)] \rightarrow \mathcal{K}_{C}^{+}$be a LR- $\chi$-preinvex $I-V \cdot F$ with $\mu<\mu+\varphi(\omega, \mu)$, such that $\mathfrak{S}(z)=\left[\mathfrak{S}_{*}(z), \mathfrak{S}^{*}(z)\right]$ for all $z \in[\mu, \mu+\varphi(\omega, \mu)]$. If $\mathfrak{S} \in L\left([\mu, \mu+\varphi(\omega, \mu)], \mathcal{K}_{C}^{+}\right)$and $\mathcal{D}:[\mu, \mu+\varphi(\omega, \mu)] \rightarrow \mathbb{R}, \mathcal{D}(z) \geq 0$, symmetric with respect to $\frac{2 \mu+\varphi(\omega, \mu)}{2}$, then:

$$
\begin{gathered}
\frac{\Gamma(\alpha)}{(\varphi(\omega, \mu))^{\alpha}}\left[\mathcal{I}_{\mu^{+}}^{\alpha} \mathfrak{S} \mathcal{D}(\mu+\varphi(\omega, \mu))+\mathcal{I}_{\mu+\varphi(\omega, \mu)^{-}}^{\alpha} \mathfrak{S D}(\mu)\right] \\
\leq_{p}(\mathfrak{S}(\mu)+\mathfrak{S}(\mu+\varphi(\omega, \mu))) \int_{0}^{1} \varsigma^{\alpha-1}[\chi(\varsigma)+\chi(1-\varsigma)] D(\mu+\varsigma \varphi(\omega, \mu)) d \varsigma .
\end{gathered}
$$

If $\mathfrak{S}$ is pre-incave $I-V \cdot F$, then inequality (26) is reversed.

Proof. Let $\mathfrak{S}$ be a LR- $\chi$-pre-invex $I-V \cdot F$ and $\varsigma^{\alpha-1} \mathcal{D}(\mu+(1-\varsigma) \varphi(\omega, \mu)) \geq 0$. Then, we have:

$$
\begin{aligned}
& \varsigma^{\alpha-1} \mathfrak{S}_{*}(\mu+(1-\varsigma) \varphi(\omega, \mu)) \mathcal{D}(\mu+(1-\varsigma) \varphi(\omega, \mu)) \\
& \leq \varsigma^{\alpha-1}\left(\chi(\varsigma) \mathfrak{S}_{*}(\mu)+\chi(1-\varsigma) \mathfrak{S}_{*}(\mu+\varphi(\omega, \mu))\right) \mathcal{D}(\mu+(1-\varsigma) \varphi(\omega, \mu)) \\
& \varsigma^{\alpha-1} \mathfrak{S}^{*}(\mu+(1-\varsigma) \varphi(\omega, \mu)) \mathcal{D}(\mu+(1-\varsigma) \varphi(\omega, \mu)) \\
& \quad \leq \varsigma^{\alpha-1}\left(\chi(\varsigma) \mathfrak{S}^{*}(\mu)+\chi(1-\varsigma) \mathfrak{S}^{*}(\mu+\varphi(\omega, \mu))\right) \mathcal{D}(\mu+(1-\varsigma) \varphi(\omega, \mu)),
\end{aligned}
$$

and:

$$
\begin{aligned}
& \varsigma^{\alpha-1} \mathfrak{S}_{*}(\mu+\varsigma \varphi(\omega, \mu)) \mathcal{D}(\mu+\varsigma \varphi(\omega, \mu)) \\
& \leq \varsigma^{\alpha-1}\left(\chi(1-\varsigma) \mathfrak{S}_{*}(\mu)+\chi(\varsigma) \mathfrak{S}_{*}(\mu+\varphi(\omega, \mu))\right) \mathcal{D}(\mu+\varsigma \varphi(\omega, \mu)) \\
& \varsigma^{\alpha-1} \mathfrak{S}^{*}(\mu+\varsigma \varphi(\omega, \mu)) \mathcal{D}(\mu+\varsigma \varphi(\omega, \mu)) \\
& \quad \leq \varsigma^{\alpha-1}\left(\chi(1-\varsigma) \mathfrak{S}^{*}(\mu)+\chi(\varsigma) \mathfrak{S}^{*}(\mu+\varphi(\omega, \mu))\right) \mathcal{D}(\mu+\varsigma \varphi(\omega, \mu)) .
\end{aligned}
$$

After adding (27) and (28), and integrating over [0, 1], we get: 


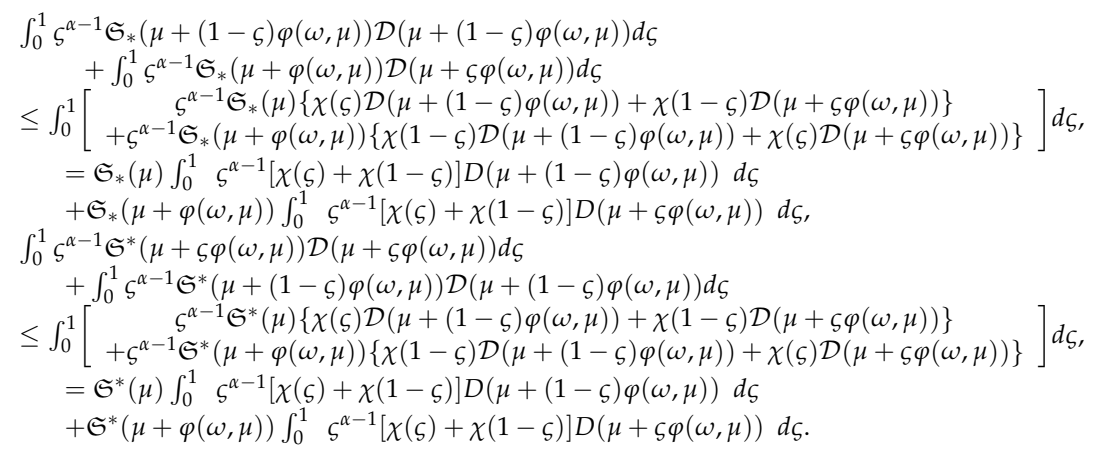

Taking the right hand side of inequality (29), we have:

$$
\begin{gathered}
\int_{0}^{1} \varsigma^{\alpha-1} \mathfrak{S}_{*}(\mu+(1-\varsigma) \varphi(\omega, \mu)) \mathcal{D}(\mu+\varsigma \varphi(\omega, \mu)) d \varsigma \\
+\int_{0}^{1} \varsigma^{\alpha-1} \mathfrak{S}_{*}(\mu+\varsigma \varphi(\omega, \mu)) \mathcal{D}(\mu+\varsigma \varphi(\omega, \mu)) d \varsigma \\
=\frac{1}{(\varphi(\omega, \mu))^{\alpha}} \int_{\mu}^{\mu+\varphi(\omega, \mu)}(z-\mu)^{\alpha-1} \mathfrak{S}_{*}(2 \mu+\varphi(\omega, \mu)-z) \mathcal{D}(z) d z \\
+\frac{1}{(\varphi(\omega, \mu))^{\alpha}} \int_{\mu}^{\mu+\varphi(\omega, \mu)}(z-\mu)^{\alpha-1} \mathfrak{S}_{*}(z) \mathcal{D}(z) d z \\
=\frac{1}{(\varphi(\omega, \mu))^{\alpha}} \int_{\mu}^{\mu+\varphi(\omega, \mu)}(\mu+\varphi(\omega, \mu)-z)^{\alpha-1} \mathfrak{S}_{*}(z) \mathcal{D}(2 \mu+\varphi(\omega, \mu)-z) d z \\
\quad+\frac{1}{(\varphi(\omega, \mu))^{\alpha}} \int_{\mu}^{\mu+\varphi(\omega, \mu)}(z-\mu)^{\alpha-1} \mathfrak{S}_{*}(z) \mathcal{D}(z) d z \\
=\frac{\Gamma(\alpha)}{(\varphi(\omega, \mu))^{\alpha}}\left[\mathcal{I}_{\mu^{+}}^{\alpha} \mathfrak{S}_{*} \mathcal{D}(\mu+\varphi(\omega, \mu))+\mathcal{I}_{\mu+\varphi(\omega, \mu)^{-}} \mathfrak{S}_{*} \mathcal{D}(\mu)\right], \\
\int_{0}^{1} \varsigma^{\alpha-1} \mathfrak{S}^{*}(\mu+(1-\varsigma) \varphi(\omega, \mu)) \mathcal{D}(\mu+\varsigma \varphi(\omega, \mu)) d \varsigma \\
+\int_{0}^{1} \varsigma^{\alpha-1} \mathfrak{S}^{*}(\mu+\varsigma \varphi(\omega, \mu)) \mathcal{D}(\mu+\varsigma \varphi(\omega, \mu)) d \varsigma \\
\frac{\Gamma(\alpha)}{(\varphi(\omega, \mu))^{\alpha}}\left[\mathcal{I}_{\mu^{+}}^{\alpha} \mathfrak{S}^{*} \mathcal{D}(\mu+\varphi(\omega, \mu))+\mathcal{I}_{\mu+\varphi(\omega, \mu)^{-}}^{\alpha} \mathfrak{S}^{*} \mathcal{D}(\mu)\right]
\end{gathered}
$$

From (30), we have:

$$
\begin{aligned}
\frac{\Gamma(\alpha)}{(\varphi(\omega, \mu))^{\alpha}} & {\left[\mathcal{I}_{\mu^{+}}^{\alpha} \mathfrak{S}_{*} \mathcal{D}(\mu+\varphi(\omega, \mu))+\mathcal{I}_{\mu+\varphi(\omega, \mu)^{\alpha}}^{\alpha} \mathfrak{S}_{*} \mathcal{D}(\mu)\right] } \\
& \leq\left(\mathfrak{S}_{*}(\mu)+\mathfrak{S}_{*}(\mu+\varphi(\omega, \mu))\right) \int_{0}^{1} \varsigma^{\alpha-1}\left[\begin{array}{c}
\chi(\varsigma) \\
+\chi(1-\varsigma)
\end{array}\right] D(\mu+\varsigma \varphi(\omega, \mu)) \\
\frac{\Gamma(\alpha)}{(\varphi(\omega, \mu))^{\alpha}} & {\left[\mathcal{I}_{\mu^{+}}^{\alpha} \mathfrak{S}^{*} \mathcal{D}(\mu+\varphi(\omega, \mu))+\mathcal{I}_{\mu+\varphi(\omega, \mu)^{\alpha}}^{\alpha} \mathfrak{S}^{*} \mathcal{D}(\mu)\right] } \\
& \leq\left(\mathfrak{S}^{*}(\mu)+\mathfrak{S}^{*}(\mu+\varphi(\omega, \mu))\right) \int_{0}^{1} \varsigma^{\alpha-1}\left[\begin{array}{c}
\chi(\varsigma) \\
+\chi(1-\varsigma)
\end{array}\right] D(\mu+\varsigma \varphi(\omega, \mu)),
\end{aligned}
$$

that is:

$$
\begin{aligned}
& \quad \frac{\Gamma(\alpha)}{(\varphi(\omega, \mu))^{\alpha}}\left[\mathcal{I}_{\mu^{+}}^{\alpha} \mathfrak{S}_{*} \mathcal{D}(\mu+\varphi(\omega, \mu))+\mathcal{I}_{\mu+\varphi(\omega, \mu)^{-}}^{\alpha *} \mathfrak{S}_{*} \mathcal{D}(\mu), \mathcal{I}_{\mu^{+}}^{\alpha} \mathfrak{S}^{*} \mathcal{D}(\mu+\varphi(\omega, \mu))+\mathcal{I}_{\mu+\varphi(\omega, \mu)^{-}}^{\alpha} \mathfrak{S}^{*} \mathcal{D}(\mu)\right] \\
& \leq_{p}\left[\mathfrak{S}_{*}(\mu)+\mathfrak{S}_{*}(\mu+\varphi(\omega, \mu)), \mathfrak{S}^{*}(\mu)+\mathfrak{S}^{*}(\mu+\varphi(\omega, \mu))\right] \int_{0}^{1} \varsigma^{\alpha-1}[\chi(\varsigma)+\chi(1-\varsigma)] D(\mu+\varsigma \varphi(\omega, \mu)) d \varsigma
\end{aligned}
$$

Hence,

$$
\begin{gathered}
\frac{\Gamma(\alpha)}{(\varphi(\omega, \mu))^{\alpha}}\left[\mathcal{I}_{\mu^{+}}^{\alpha} \mathfrak{S} \mathcal{D}(\mu+\varphi(\omega, \mu))+\mathcal{I}_{\mu+\varphi(\omega, \mu)^{-}} \mathfrak{S} \mathcal{D}(\mu)\right] \\
\leq_{p}(\mathfrak{S}(\mu)+\mathfrak{S}(\omega)) \int_{0}^{1} \varsigma^{\alpha-1}[\chi(\varsigma)+\chi(1-\varsigma)] D(\mu+\varsigma \varphi(\omega, \mu)) d \varsigma
\end{gathered}
$$

Now, we propose the first $H-H$ Fejér inequality for LR--pre-invex $I-V \cdot F$ using the interval Riemann-Liouville fractional integral. Then we will prove the validity of Theorem 6 and Theorem 7 with a nontrivial Example 3. 
Theorem 7. (First fractional $H-H$ Fejér inequality) Let $\mathfrak{S}:[\mu, \mu+\varphi(\omega, \mu)] \rightarrow \mathcal{K}_{C}^{+}$be a LR--preinvex $I-V \cdot F$ such that $\mathfrak{S}(z)=\left[\mathfrak{S}_{*}(z), \mathfrak{S}^{*}(z)\right]$ for all $z \in[\mu, \mu+\varphi(\omega, \mu)]$. Let $\mathfrak{S} \in L\left([\mu, \mu+\varphi(\omega, \mu)], \mathcal{K}_{C}^{+}\right)$and $\mathcal{D}:[\mu, \mu+\varphi(\omega, \mu)] \rightarrow \mathbb{R}, \mathcal{D}(z) \geq 0$, symmetric with respect to $\frac{2 \mu+\varphi(\omega, \mu)}{2}$. If $\varphi$ satisfies condition $C$, then:

$$
\begin{aligned}
\frac{1}{2 \chi\left(\frac{1}{2}\right)} & \mathfrak{S}\left(\frac{2 \mu+\varphi(\omega, \mu)}{2}\right)\left[\mathcal{I}_{\mu^{+}}^{\alpha} \mathcal{D}(\mu+\varphi(\omega, \mu))+\mathcal{I}_{\mu+\varphi(\omega, \mu)^{-}}^{\alpha} \mathcal{D}(\mu)\right] \\
& \leq p\left[\mathcal{I}_{\mu^{+}}^{\alpha} \mathfrak{S D}(\mu+\varphi(\omega, \mu))+\mathcal{I}_{\mu+\varphi(\omega, \mu)^{-}}^{\alpha} \mathfrak{S D}(\mu)\right]
\end{aligned}
$$

If $\mathfrak{S}$ is pre-incave $I-V \cdot F$, then inequality (31) is reversed.

Proof. Since $\mathfrak{S}$ is a LR- $\chi$-pre-invex $I-V \cdot F$ then, we have:

$$
\begin{aligned}
& \mathfrak{S}_{*}\left(\frac{2 \mu+\varphi(\omega, \mu)}{2}\right) \leq \chi\left(\frac{1}{2}\right)\left(\mathfrak{S}_{*}(\mu+(1-\varsigma) \varphi(\omega, \mu))+\mathfrak{S}_{*}(\mu+\varsigma \varphi(\omega, \mu))\right) \\
& \mathfrak{S}^{*}\left(\frac{2 \mu+\varphi(\omega, \mu)}{2}\right) \leq \chi\left(\frac{1}{2}\right)\left(\mathfrak{S}^{*}(\mu+(1-\varsigma) \varphi(\omega, \mu))+\mathfrak{S}^{*}(\mu+\varsigma \varphi(\omega, \mu))\right) .
\end{aligned}
$$

Since $\mathcal{D}(\mu+(1-\varsigma) \varphi(\omega, \mu))=\mathcal{D}(\mu+\varsigma \varphi(\omega, \mu))$, then by multiplying (32) by $\varsigma^{\alpha-1} \mathcal{D}(\mu+\varsigma \varphi(\omega, \mu))$ and integrate it with respect to $\varsigma$ over $[0,1]$, we obtain:

$$
\begin{aligned}
& \mathfrak{S}_{*}\left(\frac{2 \mu+\varphi(\omega, \mu)}{2}\right) \int_{0}^{1} \varsigma^{\alpha-1} \mathcal{D}(\mu+\varsigma \varphi(\omega, \mu)) d \varsigma \\
& \leq \chi\left(\frac{1}{2}\right)\left(\begin{array}{c}
\int_{0}^{1} \varsigma^{\alpha-1} \mathfrak{S}_{*}(\mu+(1-\varsigma) \varphi(\omega, \mu)) \mathcal{D}(\mu+\varsigma \varphi(\omega, \mu)) d \varsigma \\
+\int_{0}^{1} \varsigma^{\alpha-1} \mathfrak{S}_{*}(\mu+\varsigma \varphi(\omega, \mu)) \mathcal{D}(\mu+\varsigma \varphi(\omega, \mu)) d \varsigma
\end{array}\right), \\
& \mathfrak{S}^{*}\left(\frac{2 \mu+\varphi(\omega, \mu)}{2}\right) \int_{0}^{1} \varsigma^{\alpha-1} \mathcal{D}(\mu+\varsigma \varphi(\omega, \mu)) d \varsigma \\
& \leq \chi\left(\frac{1}{2}\right)\left(\begin{array}{c}
\int_{0}^{1} \varsigma^{\alpha-1} \mathfrak{S}^{*}(\mu+(1-\varsigma) \varphi(\omega, \mu)) \mathcal{D}(\mu+\varsigma \varphi(\omega, \mu)) d \varsigma \\
+\int_{0}^{1} \varsigma^{\alpha-1} \mathfrak{S}^{*}(\mu+\varsigma \varphi(\omega, \mu)) \mathcal{D}(\mu+\varsigma \varphi(\omega, \mu)) d \varsigma
\end{array}\right) .
\end{aligned}
$$

Let $x=\mu+\varsigma \varphi(\omega, \mu)$. Then, on the right hand side of inequality (33), we have:

$$
\begin{aligned}
\int_{0}^{1} \varsigma^{\alpha-1} \mathfrak{S}_{*}(\mu+(1-\varsigma) \varphi(\omega, \mu)) \mathcal{D}(\mu+\varsigma \varphi(\omega, \mu)) d \varsigma \\
\quad+\int_{0}^{1} \varsigma^{\alpha-1} \mathfrak{S}_{*}(\mu+\varsigma \varphi(\omega, \mu)) \mathcal{D}(\mu+\varsigma \varphi(\omega, \mu)) d \varsigma \\
=\frac{1}{(\varphi(\omega, \mu))^{\alpha}} \int_{\mu}^{\mu+\varphi(\omega, \mu)}(z-\mu)^{\alpha-1} \mathfrak{S}_{*}(2 \mu+\varphi(\omega, \mu)-z) \mathcal{D}(z) d z \\
\quad+\frac{1}{(\varphi(\omega, \mu))^{\alpha}} \int_{\mu}^{\mu+\varphi(\omega, \mu)}(z-\mu)^{\alpha-1} \mathfrak{S}_{*}(z) \mathcal{D}(z) d z \\
=\frac{1}{(\varphi(\omega, \mu))^{\alpha}} \int_{\mu}^{\mu+\varphi(\omega, \mu)}(z-\mu)^{\alpha-1} \mathfrak{S}_{*}(z) \mathcal{D}(\mu-\omega-z) d z \\
\quad+\frac{1}{(\varphi(\omega, \mu))^{\alpha}} \int_{\mu}^{\mu+\varphi(\omega, \mu)}(z-\mu)^{\alpha-1} \mathfrak{S}_{*}(z) \mathcal{D}(z) d z \\
\left.=\frac{\Gamma(\alpha)}{(\varphi(\omega, \mu))^{\alpha}} \mathcal{I}_{\mu^{+}}^{\alpha} \mathfrak{S}_{*} \mathcal{D}(\mu+\varphi(\omega, \mu))+\mathcal{I}_{\mu+\varphi(\omega, \mu)^{-}}^{\alpha} \mathfrak{S}_{*} \mathcal{D}(\mu)\right], \\
\int_{0}^{1} \varsigma^{\alpha-1} \mathfrak{S}^{*}\left(\mu+\left(1-\varsigma^{2}\right) \varphi(\omega, \mu)\right) \mathcal{D}(\mu+\varsigma \varphi(\omega, \mu)) d \varsigma \\
\quad+\int_{0}^{1} \varsigma^{\alpha-1} \mathfrak{S}^{*}(\mu+\varsigma \varphi(\omega, \mu)) \mathcal{D}(\mu+\varsigma \varphi(\omega, \mu)) d \varsigma \\
=\frac{\Gamma(\alpha)}{(\varphi(\omega, \mu))^{\alpha}}\left[\mathcal{I}_{\mu^{+}}^{\alpha} \mathfrak{S}^{*} \mathcal{D}(\mu+\varphi(\omega, \mu))+\mathcal{I}_{\mu+\varphi(\omega, \mu)^{-}} \mathfrak{S}^{*} \mathcal{D}(\mu)\right] .
\end{aligned}
$$

Then from (34), we have:

$$
\begin{array}{r}
\frac{1}{2 \chi\left(\frac{1}{2}\right)} \mathfrak{S}_{*}\left(\frac{2 \mu+\varphi(\omega, \mu)}{2}\right)\left[\mathcal{I}_{\mu^{+}}^{\alpha} \mathcal{D}(\mu+\varphi(\omega, \mu))+\mathcal{I}_{\mu+\varphi(\omega, \mu)^{-}}^{\alpha} \mathcal{D}(\mu)\right] \\
\leq\left[\mathcal{I}_{\mu^{+}}^{\alpha} \mathfrak{S}_{*} \mathcal{D}(\mu+\varphi(\omega, \mu))+\mathcal{I}_{\mu+\varphi(\omega, \mu)^{-}}^{\alpha} \mathfrak{S}_{*} \mathcal{D}(\mu)\right] \\
\frac{1}{2 \chi\left(\frac{1}{2}\right)} \mathfrak{S}^{*}\left(\frac{2 \mu+\varphi(\omega, \mu)}{2}\right)\left[\mathcal{I}_{\mu^{+}}^{\alpha} \mathcal{D}(\mu+\varphi(\omega, \mu))+\mathcal{I}_{\mu+\varphi(\omega, \mu)^{-}}^{\alpha} \mathcal{D}(\mu)\right] \\
\leq\left[\mathcal{I}_{\mu^{+}}^{\alpha} \mathfrak{S}^{*} \mathcal{D}(\mu+\varphi(\omega, \mu))+\mathcal{I}_{\mu+\varphi(\omega, \mu)^{-}}^{\alpha} \mathfrak{S}^{*} \mathcal{D}(\mu)\right],
\end{array}
$$

from which, we have: 


$$
\begin{gathered}
\frac{1}{2 \chi\left(\frac{1}{2}\right)}\left[\mathfrak{S}_{*}\left(\frac{2 \mu+\varphi(\omega, \mu)}{2}\right), \mathfrak{S}^{*}\left(\frac{2 \mu+\varphi(\omega, \mu)}{2}\right)\right]\left[\mathcal{I}_{\mu^{+}}^{\alpha} \mathcal{D}(\mu+\varphi(\omega, \mu))+\mathcal{I}_{\mu+\varphi(\omega, \mu)^{-}}^{\alpha} \mathcal{D}(\mu)\right] \\
\leq p\left[\mathcal{I}_{\mu^{+}}^{\alpha} \mathfrak{S}_{*} \mathcal{D}(\mu+\varphi(\omega, \mu))+\mathcal{I}_{\mu+\varphi(\omega, \mu)^{-}}^{\alpha} \mathfrak{S}_{*} \mathcal{D}(\mu), \mathcal{I}_{\mu^{+}}^{\alpha} \mathfrak{S}^{*} \mathcal{D}(\mu+\varphi(\omega, \mu))+\mathcal{I}_{\mu+\varphi(\omega, \mu)^{-}}^{\alpha} \mathfrak{S}^{*} \mathcal{D}(\mu)\right], \\
\text { and it follows that: } \\
\frac{1}{2 \chi\left(\frac{1}{2}\right)} \mathfrak{S}\left(\frac{2 \mu+\varphi(\omega, \mu)}{2}\right)\left[\mathcal{I}_{\mu^{+}}^{\alpha} \mathcal{D}(\mu+\varphi(\omega, \mu))+\mathcal{I}_{\mu+\varphi(\omega, \mu)^{-}}^{\alpha} \mathcal{D}(\mu)\right] \\
\leq p_{p}\left[\mathcal{I}_{\mu^{+}}^{\alpha} \mathfrak{S D}(\mu+\varphi(\omega, \mu))+\mathcal{I}_{\mu+\varphi(\omega, \mu)^{-}} \mathfrak{S D D}^{\alpha}(\mu)\right]
\end{gathered}
$$

This completes the proof.

Remark 5. If $\mathcal{D}(z)=1$, then from (26) and (31), we get Theorem 3.

If $\chi(\varsigma)=\varsigma$, then from (26) and (31), we achieve the following coming inequality, see [42]:

$$
\begin{gathered}
\mathfrak{S}\left(\frac{2 \mu+\varphi(\omega, \mu)}{2}\right)\left[\mathcal{I}_{\mu^{+}}^{\alpha} \mathcal{D}(\mu+\varphi(\omega, \mu))+\mathcal{I}_{\mu+\varphi(\omega, \mu)^{-}}^{\alpha} \mathcal{D}(\mu)\right] \\
\leq_{p}\left[\mathcal{I}_{\mu^{+}}^{\alpha} \mathfrak{S} \mathcal{D}\left(\mu+\{\varphi(\omega, \mu))+\mathcal{I}_{\mu+\varphi(\omega, \mu)^{-}}^{\alpha} \mathfrak{S} \mathcal{D}(\mu)\right]\right. \\
\leq_{p} \frac{\mathfrak{S}(\mu)+\mathfrak{S}(\mu+\varphi(\omega, \mu))}{2}\left[\mathcal{I}_{\mu^{+}}^{\alpha} \mathcal{D}(\mu+\varphi(\omega, \mu))+\mathcal{I}_{\mu+\varphi(\omega, \mu)^{-}}^{\alpha} \mathcal{D}(\mu)\right] \\
\leq_{p} \frac{\mathfrak{S}(\mu)+\mathfrak{S}(\omega)}{2}\left[\mathcal{I}_{\mu^{+}}^{\alpha} \mathcal{D}(\mu+\varphi(\omega, \mu))+\mathcal{I}_{\mu+\varphi(\omega, \mu)^{-}}^{\alpha} \mathcal{D}(\mu)\right]
\end{gathered}
$$

Let $\chi(\varsigma)=\varsigma$ and $\alpha=1$. Then, from (26) and (31), we achieve the following coming inequality:

$$
\mathfrak{S}\left(\frac{2 \mu+\varphi(\omega, \mu)}{2}\right) \leq_{p} \frac{1}{\int_{\mu}^{\mu+\varphi(\omega, \mu)} \mathcal{D}(z) d z}(F R) \int_{\mu}^{\mu+\varphi(\omega, \mu)} \mathfrak{S}(z) \mathcal{D}(z) d z \leq_{p} \frac{\mathfrak{S}(\mu)+\mathfrak{S}(\omega)}{2}
$$

If $\mathfrak{S}_{*}(z)=\mathfrak{S}^{*}(z)$ and $\chi(\varsigma)=\varsigma$, then from (26) and (31), we achieve the following coming inequality:

$$
\begin{gathered}
\mathfrak{S}\left(\frac{2 \mu+\varphi(\omega, \mu)}{2}\right)\left[\mathcal{I}_{\mu^{+}}^{\alpha} \mathcal{D}(\omega)+\mathcal{I}_{\omega^{-}}^{\alpha} \mathcal{D}(\mu)\right] \leq\left[\mathcal{I}_{\mu^{+}}^{\alpha} \mathfrak{S} \mathcal{D}(\mu+\varphi(\omega, \mu))+\mathcal{I}_{\mu+\varphi(\omega, \mu)^{-}}^{\alpha} \mathfrak{S} \mathcal{D}(\mu)\right] \\
\leq \frac{\mathfrak{S}(\mu)+\mathfrak{S}(\mu+\varphi(\omega, \mu))}{2}\left[\mathcal{I}_{\mu^{+}}^{\alpha} \mathcal{D}(\mu+\varphi(\omega, \mu))+\mathcal{I}_{\mu+\varphi(\omega, \mu)^{-}}^{\alpha} \mathcal{D}(\mu)\right] \\
\leq \frac{\mathfrak{S}(\mu)+\mathfrak{S}(\omega)}{2}\left[\mathcal{I}_{\mu^{+}}^{\alpha} \mathcal{D}(\mu+\varphi(\omega, \mu))+\mathcal{I}_{\mu+\varphi(\omega, \mu)^{-}}^{\alpha} \mathcal{D}(\mu)\right]
\end{gathered}
$$

If $\mathfrak{S}_{*}(z)=\mathfrak{S}^{*}(z)$ and $\alpha=1$ and $\chi(\varsigma)=\varsigma$, then from (26) and (31), we acquire the classical $H-H$ Fejér inequality.

If $\mathfrak{S}_{*}(z)=\mathfrak{S}^{*}(z)$ and $\mathcal{D}(z)=\alpha=1$ and $\chi(\varsigma)=\varsigma$, then from (26) and (31), we acquire the classical H-H inequality.

Example 3. We consider the $I-V \cdot F \mathfrak{S}:[0, \varphi(2,0)] \rightarrow \mathcal{K}_{C}^{+}$defined by, $\mathfrak{S}(z)=[1,2](2-\sqrt{z})$. Since end-point functions $\mathfrak{S}_{*}(z), \mathfrak{S}^{*}(z)$ are $L R-\chi$-pre-invex functions, then $\mathfrak{S}(z)$ is LR- $\chi$-pre-invex $I-V \cdot F$.

If:

$$
\mathcal{D}(z)=\left\{\begin{array}{cl}
\sqrt{z}, & \sigma \in[0,1] \\
\sqrt{2-z}, & \sigma \in(1,2],
\end{array}\right.
$$

then $\mathcal{D}(2-z)=\mathcal{D}(z) \geq 0$, for all $z \in[0,2]$. Since $\mathfrak{S}_{*}(z)=(2-\sqrt{z})$ and $\mathfrak{S}^{*}(z)=2(2-\sqrt{z})$. If $\chi(\varsigma)=\varsigma$ and $\alpha=\frac{1}{2}$, then we compute the following:

$$
\begin{gathered}
\frac{\mathfrak{S}_{*}(\mu)+\mathfrak{S}_{*}(\mu+\varphi(\omega, \mu))}{2} \int_{0}^{1} \varsigma^{\alpha-1}[\chi(\varsigma)+\chi(1-\varsigma)] D(\mu+\varsigma \varphi(\omega, \mu))=\frac{\pi}{\sqrt{2}}\left(\frac{4-\sqrt{2}}{2}\right), \\
\frac{\mathfrak{S}^{*}(\mu)+\mathfrak{S}^{*}(\mu+\varphi(\omega, \mu))}{2} \int_{0}^{1} \varsigma^{\alpha-1}[\chi(\varsigma)+\chi(1-\varsigma)] D(\mu+\varsigma \varphi(\omega, \mu))=\frac{\pi}{\sqrt{2}}(4-\sqrt{2}),
\end{gathered}
$$




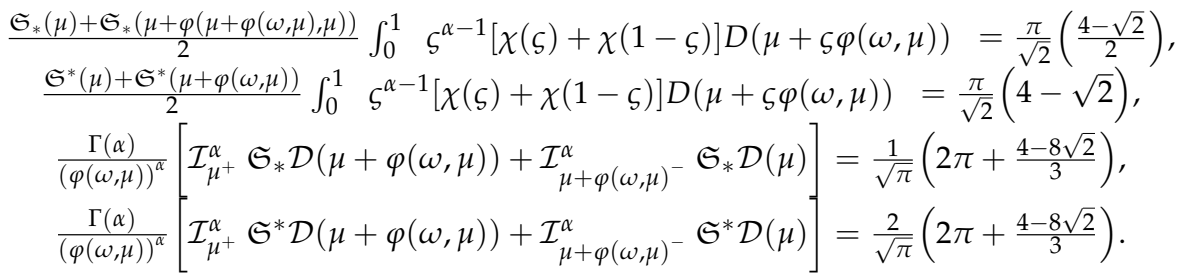

From (38) and (39), we have:

$$
\frac{1}{\sqrt{\pi}}\left[\left(2 \pi+\frac{4-8 \sqrt{2}}{3}\right), 2\left(2 \pi+\frac{4-8 \sqrt{2}}{3}\right)\right] \leq p \frac{\pi}{\sqrt{2}}\left[\frac{4-\sqrt{2}}{2}, 4-\sqrt{2}\right] .
$$

Hence, Theorem 6 is verified.

For Theorem 7, we have:

$$
\begin{aligned}
& \mathcal{I}_{\mu^{+}}^{\alpha} \mathfrak{S}_{*} \mathcal{D}(\mu+\varphi(\omega, \mu))+\mathcal{I}_{\mu+\varphi(\omega, \mu)^{-}}^{\alpha} \mathfrak{S}_{*} \mathcal{D}(\mu) \\
& \quad=\frac{1}{\sqrt{\pi}} \int_{0}^{2}(2-z)^{\frac{-1}{2}} \mathcal{D}(z)(2-\sqrt{z}) d z+\frac{1}{\sqrt{\pi}} \int_{0}^{2}(z)^{\frac{-1}{2}} \mathcal{D}(z)(2-\sqrt{z}) d z \\
& \quad=\frac{1}{\sqrt{\pi}}\left(\pi+\frac{8-8 \sqrt{2}}{3}\right)+\frac{1}{\sqrt{\pi}}\left(\pi-\frac{4}{3}\right)=\frac{1}{\sqrt{\pi}}\left(2 \pi+\frac{4-8 \sqrt{2}}{3}\right) \\
& \mathcal{I}_{\mu^{+}}^{\alpha} \mathfrak{S}^{*} \mathcal{D}(\mu+\varphi(\omega, \mu))+\mathcal{I}_{\mu+\varphi(\omega, \mu)^{-}}^{\alpha} \mathfrak{S}^{*} \mathcal{D}(\mu) \\
& \quad=\frac{2}{\sqrt{\pi}} \int_{0}^{2}(2-z)^{\frac{-1}{2}} \mathcal{D}(z)(2-\sqrt{z}) d z+\frac{2}{\sqrt{\pi}} \int_{0}^{2}(z)^{\frac{-1}{2}} \mathcal{D}(z)(2-\sqrt{z}) d z \\
& \quad=\frac{2}{\sqrt{\pi}}\left(\pi+\frac{8-8 \sqrt{2}}{3}\right)+\frac{2}{\sqrt{\pi}}\left(\pi-\frac{4}{3}\right)=\frac{2}{\sqrt{\pi}}\left(2 \pi+\frac{4-8 \sqrt{2}}{3}\right) . \\
& \frac{1}{2 \chi\left(\frac{1}{2}\right)} \mathfrak{S}_{*}\left(\frac{2 \mu+\varphi(\omega, \mu)}{2}\right)\left[\mathcal{I}_{\mu^{+}}^{\alpha} \mathcal{D}(\mu+\varphi(\omega, \mu))+\mathcal{I}_{\mu+\varphi(\omega, \mu)^{-}}^{\alpha} \mathcal{D}(\mu)\right]=\sqrt{\pi} \\
& \frac{1}{2 \chi\left(\frac{1}{2}\right)} \mathfrak{S}^{*}\left(\frac{2 \mu+\varphi(\omega, \mu)}{2}\right)\left[\mathcal{I}_{\mu^{+}}^{\alpha} \mathcal{D}(\mu+\varphi(\omega, \mu))+\mathcal{I}_{\mu+\varphi(\omega, \mu)^{-}}^{\alpha} \mathcal{D}(\mu)\right]=2 \sqrt{\pi} .
\end{aligned}
$$

\section{Conclusions}

We have proposed the class of LR- $\chi$-pre-invexity for $I-V \cdot F s$. By using this class, we have presented several interval $H-H$ inequalities and interval $H-H$ Fejér inequalities using interval Riemann-Liouville fractional integral operators. Useful examples that illustrate the applicability of theory developed in this study are also presented. In future, we intend to discuss generalized LR- $\chi$-pre-invex $I-V \cdot F$ s. We hope that this concept will be helpful for other authors to play their roles in different fields of sciences.

Author Contributions: Conceptualization, M.B.K.; methodology, M.B.K.; validation, S.T., M.S.S. and H.G.Z.; formal analysis, K.N.; investigation, M.S.S.; resources, S.T.; data curation, H.G.Z.; writingoriginal draft preparation, M.B.K., K.N. and H.G.Z.; writing-review and editing, M.B.K. and S.T.; visualization, H.G.Z.; supervision, M.B.K. and M.S.S.; project administration, M.B.K.; funding acquisition, K.N., M.S.S. and H.G.Z. All authors have read and agreed to the published version of the manuscript.

Funding: This research received no external funding.

Institutional Review Board Statement: Not applicable.

Informed Consent Statement: Not applicable.

Acknowledgments: The authors would like to thank the Rector, COMSATS University Islamabad, Islamabad, Pakistan, for providing excellent research. This work was funded by Taif University Researchers Supporting Project number (TURSP-2020/345), Taif University, Taif, Saudi Arabia. Moreover, this research has also received funding support from the National Science, Research and Innovation Fund (NSRF), Thailand.

Conflicts of Interest: The authors declare no conflict of interest. 


\section{References}

1. Hermite, C. Sur deux limites d'une intégrale définie. Mathesis 1883, 3, 82-97.

2. Hadamard, J. Étude sur les propriétés des fonctions entières et en particulier d'une fonction considérée par Riemann. J. Mathématiques Pures Appliquées 1893, 7, 171-215.

3. Dragomir, S.; Pearce, C. Selected Topics on Hermite-Hadamard Inequalities and Applications; RGMIA Monographs; Victoria University: Melbourne, Australia, 2004.

4. Peajcariaac, J.E.; Tong, Y.L. Convex Functions, Partial Orderings, and Statistical Applications; Academic Press: Bostan, MA, USA, 1992.

5. Chen, F. A note on Hermite-Hadamard inequalities for products of convex functions. J. Appl. Math. 2013, 2013, 1-6. [CrossRef]

6. Dragomir, S.S. Inequalities of Hermite-Hadamard type for h-convex functions on linear spaces. Proyecciones 2015 , 34, 323-341. [CrossRef]

7. Dragomir, S.S. Two mappings in connection to Hadamard's inequalities. J. Math. Anal. Appl. 1992, 167, 49-56. [CrossRef]

8. Dragomir, S.J.; Pecaric, L.-E. Persson, Some inequalities of Hadamard type. Soochow J. Math. 1995, $21,335-341$.

9. Pachpatte, B. On some inequalities for convex functions. RGMIA Res. Rep. Collect. 2003, 6, 1-9.

10. Wang, J.; Li, X.; Zhu, C. Refinements of Hermite-Hadamard type inequalities involving fractional integrals. Bull. Belg. Math. Soc. Simon Stevin 2013, 20, 655-666. [CrossRef]

11. Sarikaya, M.Z.; Ertugral, F. On the generalized Hermite-Hadamard inequalities. Ann. Univ. Craioval Math. Comput. Sci. Ser. 2017, $47,193-213$.

12. Sarikaya, M.; Yildirim, H. On generalization of the Riesz potential. Indian J. Math. Math. Sci. 2007, 3, 231-235.

13. Ertugral, F.; Sarikaya, M.Z. Simpson type integral inequalities for generalized fractional integral. Rev. R. Acad. Cienc. Exactas Fís. Nat. Ser. A Mat. 2019, 113, 3115-3124. [CrossRef]

14. Tseng, K.L.; Hwang, S.R. New Hermite-Hadamard-type inequalities and their applications. Filomat 2016, 30, 3667-3680. [CrossRef]

15. Moore, R.E. Interval Analysis; Prentice Hall: Englewood Cliffs, NJ, USA, 1966.

16. Chalco-Cano, Y.; Flores-Franulic, A.; Román-Flores, H. Ostrowski type inequalities for interval-valued functions using generalized Hukuhara derivative. Comput. Appl. Math. 2012, 31, 457-472.

17. Chalco-Cano, Y.; Lodwick, W.A.; Condori-Equice, W. Ostrowski type inequalities and applications in numerical integration for interval-valued functions. Soft Comput. 2015, 19, 3293-3300. [CrossRef]

18. Román-Flores, H.; Chalco-Cano, Y.; Lodwick, W. Some integral inequalities for interval-valued functions. Comput. Appl. Math. 2018, 37, 1306-1318. [CrossRef]

19. Costa, T. Jensen's inequality type integral for fuzzy-interval-valued functions. Fuzzy Sets Syst. 2017, 327, 31-47. [CrossRef]

20. Costa, T.; Román-Flores, H. Some integral inequalities for fuzzy-interval-valued functions. Inf. Sci. 2017, 420, 110-125. [CrossRef]

21. Flores-Franulic, A.; Chalco-Cano, Y.; Román-Flores, H. An Ostrowski type inequality for interval-valued functions. In Proceedings of the 2013 Joint IFSA World Congress and NAFIPS Annual Meeting (IFSA/NAFIPS), Edmonton, AB, Canada, 24-28 June 2013; pp. 1459-1462.

22. Román-Flores, H.; Chalco-Cano, Y.; Silva, G.N. A note on Gronwall type inequality for interval-valued functions. In Proceedings of the 2013 Joint IFSA World Congress and NAFIPS Annual Meeting (IFSA/NAFIPS), Edmonton, AB, Canada, 24-28 June 2013; pp. 1455-1458.

23. Sadowska, E. Hadamard inequality and a refinement of Jensen inequality for set valued functions. Results Math. 1997, 32, 332-337. [CrossRef]

24. Mitroi, F.-C.; Nikodem, K.; Wasowicz, S. Hermite-Hadamard inequalities for convex set-valued functions. Demonstr. Math. 2013, 46, 655-662. [CrossRef]

25. Nikodem, K.; Sanchez, J.L.; Sanchez, L. Jensen and Hermite-Hadamard inequalities for strongly convex set-valued maps. Math. Aeterna 2014, 4, 979-987.

26. Khan, M.B.; Noor, M.A.; Noor, K.I.; Chu, Y.M. New Hermite-Hadamard type inequalities for (h1, h2)-convex fuzzy-interval-valued functions. Adv. Differ. Equ. 2021, 2021, 6-20. [CrossRef]

27. Khan, M.B.; Mohammed, P.O.; Noor, M.A.; Hamed, Y.S. New Hermite-Hadamard inequalities in fuzzy-interval fractional calculus and related inequalities. Symmetry 2021, 13, 673. [CrossRef]

28. Khan, M.B.; Mohammed, P.O.; Noor, M.A.; Alsharif, A.M.; Noor, K.I. New fuzzy-interval inequalities in fuzzy-interval fractional calculus by means of fuzzy order relation. AIMS Math. 2021, 6, 10964-10988. [CrossRef]

29. Khan, M.B.; Noor, M.A.; Abdullah, L.; Chu, Y.M. Some new classes of preinvex fuzzy-interval-valued functions and inequalities. Int. J. Comput. Intell. Syst. 2021, 14, 1403-1418. [CrossRef]

30. Liu, P.; Khan, M.B.; Noor, M.A.; Noor, K.I. New Hermite-Hadamard and Jensen inequalities for log-s-convex fuzzy-interval-valued functions in the second sense. Complex Intell. Syst. 2021, 2021, 1-15. [CrossRef]

31. Khan, M.B.; Noor, M.A.; Al-Bayatti, H.M.; Noor, K.I. Some new inequalities for LR-log-h-convex interval-valued functions by means of pseudo order relation. Appl. Math. Inf. Sci. 2021, 15, 459-470.

32. Sana, G.; Khan, M.B.; Noor, M.A.; Mohammed, P.O.; Chu, Y.M. Harmonically convex fuzzy-interval-valued functions and fuzzy-interval Riemann-Liouville fractional integral inequalities. Int. J. Comput. Intell. Syst. 2021, 14, 1809-1822. [CrossRef]

33. Khan, M.B.; Mohammed, P.O.; Noor, M.A.; Abualnaja, K.M. Fuzzy integral inequalities on coordinates of convex fuzzy intervalvalued functions. Math. Biosci. Eng. 2021, 18, 6552-6580. [CrossRef] 
34. Niculescu, C.P.; Persson, L.E. Convex Functions and Their Applications; Springer: New York, NY, USA, 2006.

35. Zhang, D.; Guo, C.; Chen, D.; Wang, G. Jensen's inequalities for set-valued and fuzzy set-valued functions. Fuzzy Sets Syst. 2020, 2020, 1-27. [CrossRef]

36. Lupulescu, V. Fractional calculus for interval-valued functions. Fuzzy Sets Syst. 2015, 265, 63-85. [CrossRef]

37. Budak, H.; Tunç, T.; Sarikaya, M. Fractional Hermite-Hadamard-type inequalities for interval-valued functions. Proc. Am. Math. Soc. 2020, 148, 705-718. [CrossRef]

38. Khan, M.B.; Noor, M.A.; Noor, K.I. Some Inequalities for LR-( $\left.\mathrm{h}_{1}, \mathrm{~h}_{2}\right)$-Convex Interval-Valued Functions by Means of Pseudo Order Relation. Int. J. Comput. Intell. Syst. 2021, 14, 180. [CrossRef]

39. Khan, M.B.; Mohammed, P.O.; Noor, M.A.; Baleanu, D.; Guirao, J. Some New Fractional Estimates of Inequalities for LR-p-Convex Interval-Valued Functions by Means of Pseudo Order Relation. Axioms 2021, 10, 175. [CrossRef]

40. Mohan, S.R.; Neogy, S.K. On invex sets and preinvex functions. J. Math. Anal. Appl. 1995, 189, 901-908. [CrossRef]

41. Khan, M.B.; Noor, M.A.; Abdeljawad, T.; Mousa, A.A.A.; Abdalla, B.; Alghamdi, S.M. LR-Preinvex Interval-Valued Functions and Riemann-Liouville Fractional Integral Inequalities. Fractal Fract. 2021, 5, 243. [CrossRef]

42. Khan, M.B.; Treanțǎ, S.; Soliman, M.S.; Nonlaopon, K.; Zaini, H.G. Some Hadamard-Fejér Type Inequalities for LR-Convex Interval-Valued Functions. Fractal Fract. 2022, 6, 6. [CrossRef]

43. Awan, M.U.; Noor, M.A.; Mihai, M.V.; Noor, K.I. Generalized Coordinated Nonconvex Functions and Integral Inequalities. Appl. Math. 2008, 12, 337-344. [CrossRef]

44. Khan, M.B.; Srivastava, H.M.; Mohammed, P.O.; Nonlaopon, K.; Hamed, Y.S. Some new Jensen, Schur and Hermite-Hadamard inequalities for log convex fuzzy interval-valued functions. AIMS Math. 2022, 7, 4338-4358. [CrossRef]

45. Khan, M.B.; Mohammed, P.O.; Machado, J.A.T.; Guirao, J.L. Integral Inequalities for Generalized Harmonically Convex Functions in Fuzzy-Interval-Valued Settings. Symmetry 2021, 13, 2352. [CrossRef]

46. Khan, M.B.; Srivastava, H.M.; Mohammed, P.O.; Baleanu, D.; Jawa, T.M. Fuzzy-interval inequalities for generalized convex fuzzy-interval-valued functions via fuzzy Riemann integrals. AIMS Math. 2022, 7, 1507-1535. [CrossRef]

47. Awan, M.U.; Noor, M.A.; Noor, K.I. Hermite-Hadamard inequalities for exponentially convex functions. Appl. Math. Inf. Sci. 2018, 12, 405-409. [CrossRef]

48. Macías-Díaz, J.E.; Khan, M.B.; Noor, M.A.; Abd Allah, A.M.; Alghamdi, S.M. Hermite-Hadamard inequalities for generalized convex functions in interval-valued calculus. AIMS Math. 2022, 7, 4266-4292. [CrossRef]

49. Saleem, N.; Abbas, M.; Raza, Z. Optimal coincidence best approximation solution in non-Archimedean Fuzzy Metric Spaces. Iran J. Fuzzy Syst. 2016, 13, 113-124.

50. Saleem, N.; Zhou, M.; Bashir, S.; Husnine, S.M. Some new generalizations of F-contraction type mappings that weaken certain conditions on Caputo fractional type differential equations. AIMS Math. 2021, 6, 12718. [CrossRef] 